\title{
SMASHing the LMC: A Tidally Induced Warp in the Outer LMC and a Large-scale Reddening Map
}

Yumi Choi ${ }^{1,2}$ (1) , David L. Nidever ${ }^{2,3}$ (1), Knut Olsen $^{3}$ (10), Robert D. Blum ${ }^{3}$ (D) Gurtina Besla $^{1}$, Dennis Zaritsky ${ }^{1}$ (D),

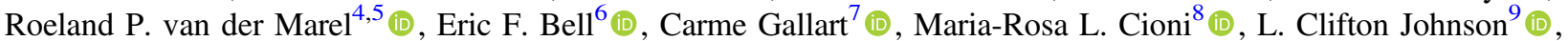
A. Katherina Vivas ${ }^{10}$ (1) , Abhijit Saha ${ }^{3}$ (1) Thomas J. L. de Boer ${ }^{11}$, Noelia E. D. Noël ${ }^{11}$, Antonela Monachesi ${ }^{12,13}$ (1), Pol Massana ${ }^{11}$,

Blair C. Conn ${ }^{14,15}$ (10), David Martinez-Delgado ${ }^{16}$ (1) , Ricardo R. Muñoz ${ }^{17}$, and Guy S. Stringfellow ${ }^{18}$ (1)

${ }^{1}$ Steward Observatory, University of Arizona, 933 North Cherry Avenue, Tucson, AZ 85721, USA; ymchoi@email.arizona.edu

2 Department of Physics, Montana State University, P.O. Box 173840, Bozeman, MT 59717-3840, USA

National Optical Astronomy Observatory, 950 North Cherry Ave, Tucson, AZ 85719, USA

${ }^{4}$ Space Telescope Science Institute, 3700 San Martin Drive, Baltimore, MD 21218, USA

${ }^{5}$ Center for Astrophysical Sciences, Department of Physics \& Astronomy, Johns Hopkins University, Baltimore, MD 21218, USA

${ }^{6}$ Department of Astronomy, University of Michigan, 1085 S. University Avenue, Ann Arbor, MI 48109-1107, USA 7 Instituto de Astrofísica de Canarias, La Laguna, Tenerife, Spain

${ }^{8}$ Leibniz-Institut für Astrophysics Potsdam (AIP), An der Sternwarte 16, D-14482 Potsdam, Germany

${ }^{9}$ Department of Physics and Astronomy, Northwestern University, 2145 Sheridan Road, Evanston, IL 60208, USA

${ }^{10}$ Cerro Tololo Inter-American Observatory, National Optical Astronomy Observatory, Casilla 603, La Serena, Chile

${ }^{11}$ Department of Physics, University of Surrey, Guildford, GU2 7XH, UK

12 Instituto de Investigación Multidisciplinario en Ciencias y Tecnología, Universidad de La Serena, Raúl Bitrán 1305, La Serena, Chile

${ }_{14}$ Departamento de Física y Astronomía, Universidad de La Serena, Av. Juan Cisternas 1200 Norte, La Serena, Chile

${ }^{14}$ Research School of Astronomy and Astrophysics, Australian National University, Canberra, ACT 2611, Australia

${ }^{15}$ Gemini Observatory, Recinto AURA, Colina El Pino s/n, La Serena, Chile

16 Astronomisches Rechen-Institut, Zentrum für Astronomie der Universität Heidelberg, Mönchhofstr. 12-14, D-69120 Heidelberg, Germany

${ }_{17}$ Departamento de Astronomía, Universidad de Chile, Camino del Observatorio 1515, Las Condes, Santiago, Chile

${ }^{18}$ Center for Astrophysics and Space Astronomy, University of Colorado, 389 UCB, Boulder, CO 80309-0389, USA Received 2018 March 22; revised 2018 September 4; accepted 2018 September 6; published 2018 October 16

\begin{abstract}
We present a study of the three-dimensional (3D) structure of the Large Magellanic Cloud (LMC) using $\sim 2.2$ million red clump (RC) stars selected from the Survey of the MAgellanic Stellar History. To correct for line-ofsight dust extinction, the intrinsic RC color and magnitude and their radial dependence are carefully measured by using internal nearly dust-free regions. These are then used to construct an accurate 2D reddening map (165 deg ${ }^{2}$ area with $\sim 10^{\prime}$ resolution) of the LMC disk and the 3D spatial distribution of RC stars. An inclined disk model is fit to the 2D distance map, yielding a best-fit inclination angle $i=25.86_{-1.39}^{+0.73}$ degrees with random errors of $\pm 0^{\circ} .19$ and line-of-nodes position angle $\theta=149.23_{-8.35}^{+6.43}$ degrees with random errors of \pm 0.49 . These angles vary with galactic radius, indicating that the LMC disk is warped and twisted likely due to the repeated tidal interactions with the Small Magellanic Cloud (SMC). For the first time, our data reveal a significant warp in the southwestern part of the outer disk starting at $\rho \sim 7^{\circ}$ that departs from the defined LMC plane up to $\sim 4 \mathrm{kpc}$ toward the SMC, suggesting that it originated from a strong interaction with the SMC. In addition, the inner disk encompassing the off-centered bar appears to be tilted up to $5^{\circ}-15^{\circ}$ relative to the rest of the LMC disk. These findings on the outer warp and the tilted bar are consistent with the predictions from the Besla et al. simulation of a recent direct collision with the SMC.
\end{abstract}

Key words: galaxies: dwarf - galaxies: interactions - galaxies: ISM - galaxies: structure - Magellanic Clouds

Supporting material: data behind figures

\section{Introduction}

The Large and Small Magellanic Clouds (LMC and SMC) are the largest satellites of the Milky Way (MW) and the closest interacting pair of dwarf galaxies. For decades it was thought that the Magellanic Clouds (MCs) had completed many orbits around the MW and that the tidal forces of the MW had been the primary mechanism that created the Magellanic Stream (e.g., Gardiner \& Noguchi 1996; Yoshizawa \& Noguchi 2003; Connors et al. 2004, 2006). However, that picture changed dramatically in the last decade as $H S T$ proper motions (Kallivayalil et al. 2006a, 2006b; Piatek et al. 2008; Kallivayalil et al. 2013) suggested that the MCs only recently fell into the MW potential (Besla et al. 2007), but that the LMC-SMC pair likely had been gravitationally bound to each other for more than several gigayears and possibly for a Hubble time
(Besla et al. 2007). The pair has also likely experienced a recent direct collision (e.g., Olsen et al. 2011; Besla et al. 2012; Noël et al. 2013; Carrera et al. 2017; Zivick et al. 2018). It thus appears that the complex morphology of the LMC disk (e.g., van der Marel 2001; Olsen \& Salyk 2002; Besla et al. 2016; Mackey et al. 2016) has more to do with the history of interactions with the SMC than with the MW. Indeed, the structure of the LMC seems to hold many keys to decoding the process of interaction between the MCs, and to their eventual fate as accreted satellites of the MW.

Many more dwarf galaxies with smaller companions will be discovered with upcoming large telescopes in the next decade especially with LSST. They will shed light on galaxy merging mechanisms. The LMC-SMC pair, however, is a unique opportunity to closely witness an ongoing hierarchical merging event (i.e., the SMC interacting with the LMC and the MCs 
merging on to the MW) and to perform detailed studies of the building process of a large galaxy like the MW.

The structure of the stellar populations and interstellar medium (ISM) across the LMC has been intensively studied, as the LMC is the closest (49.9 kpc; de Grijs et al. 2014) "laboratory" for the study of many astrophysical phenomena. The LMC has an inclined, slightly elongated, rotating, and starforming disk (Kim et al. 1998; van der Marel 2001; van der Marel \& Cioni 2001; van der Marel et al. 2002; Subramanian \& Subramaniam 2010) with a shallow stellar metallicity gradient (e.g., Carrera et al. 2008; Feast et al. 2010; Carrera et al. 2011; Piatti \& Geisler 2013; Choudhury et al. 2016; Pieres et al. 2016) and one prominent spiral arm in the central region with an off-centered bar (de Vaucouleurs \& Freeman 1972; Zhao \& Evans 2000; Zaritsky 2004; Subramanian \& Subramaniam 2009b). Its depth along the line of sight is $\sim 5 \mathrm{kpc}$ (Subramanian \& Subramaniam 2009b; Yanchulova Merica-Jones et al. 2017), and the northern outer disk shows signs of disturbance in its stellar structure (Mackey et al. 2016).

However, our understanding of the evolution of the LMC is still far from complete. This is mainly because most previous studies have heavily focused on the inner disk $\left(\lesssim 4^{\circ}\right.$ or $\lesssim 3.5 \mathrm{kpc}$ at the LMC distance) containing the majority of the ongoing star formation and ISM. Although 2MASS (Skrutskie et al. 2006) and DENIS (Epchtein et al. 1997) observed the main body of the LMC, they still go out only to $\sim 7^{\circ}$ (e.g., van der Marel 2001; van der Marel \& Cioni 2001). To understand the history of tidal interactions, it is necessary to explore the outer part of the LMC disk as well where (1) the potential is shallower and so more easily disturbed, and (2) older stellar populations are dominant and thus better trace the underlying disk structure. A good portion of the northern periphery of the LMC has been studied (e.g., Saha et al. 2010; Balbinot et al. 2015; Mackey et al. 2016), but the southern outskirt of the LMC has not been well explored.

The Survey of the MAgellanic Stellar History (SMASH) is an NOAO community Dark Energy Camera (DECam; Flaugher et al. 2015) survey of the Clouds mapping $480 \mathrm{deg}^{2}$ (distributed over $\sim 2400 \mathrm{deg}^{2}$ at $\sim 20 \%$ filling factor) to $\sim 24$ th AB mag in ugriz with the goal of identifying broadly distributed, low surface brightness stellar populations associated with the stellar halos and tidal debris of the Magellanic Clouds (Nidever et al. 2017). About one-third of the SMASH fields probe the main body of the LMC covering roughly $5^{\circ}$ north and 10.5 south of the LMC center. We note that the southern periphery of the LMC is a novel region that has been barely explored in contrast to its northern periphery.

In this study, we use red clump (RC) stars to map the dust reddening over $165 \mathrm{deg}^{2}$ and to explore the three-dimensional (3D) structure of the LMC disk. RC stars are abundant lowmass stars $\left(\lesssim 2 M_{\odot}\right.$; Castellani et al. 2000) in the core He-burning stage with intermediate age and moderately high metallicity, and constitute a prominent feature in the color-magnitude diagram (CMD). The RC stars occupy a welldefined and narrow region in the CMD since the stellar core mass at He ignition is nearly independent of their initial mass. This fundamental property results in a very limited range of effective temperatures and luminosities-making RC stars essentially "standard candles"-and allows us to accurately measure the extinctions and distances for RC stars across the LMC (Girardi 2016 and references therein). These distances are then used to create a 3D map of the LMC to study and define its structure. Many studies have used RC stars as extinction and distance probes in the LMC (e.g., Girardi \& Salaris 2001; Olsen \& Salyk 2002; Koerwer 2009; Subramanian \& Subramaniam 2009b; Haschke et al. 2011; Subramanian \& Subramaniam 2013; Tatton et al. 2013).

This paper is organized as follows. In Section 2, we briefly describe the SMASH survey and photometry data. In Section 3, we present the RC selection, maps of RC star counts, median colors, and magnitudes. Section 4 presents the measured intrinsic RC color and magnitude in the LMC and their radial profiles. Then we present and validate our reddening map in Section 5. We discuss the 3D structure of the LMC in Section 6 as well as the physical implication of our findings and explore the dependence of the resulting 3D structure on the stellar population effect in Section 7. The main conclusions are summarized in Section 8.

\section{Data and Photometry}

The SMASH survey builds on the technique first adopted by the Outer Limits Survey (Saha et al. 2010), namely, to use old mainsequence (MS) stars as tracers to reveal the relics of the formation and past interactions of the Clouds. With the large field of view of DECam, SMASH is able to use individual stars to probe down to surface brightnesses equivalent to $\Sigma_{g}=35 \mathrm{mag} \mathrm{arcsec}^{-2}$ over a vast area.

Figure 1 shows the footprints of the SMASH survey fields in the region of the MCs over the HIgas map of McClureGriffiths et al. (2009). Nidever et al. (2017) describes in detail the SMASH survey strategy, data reduction, and calibration as well as the first public data release containing $\sim 700$ million measurements of $\sim 100$ million objects in 61 deep and fully calibrated fields via the NOAO Data Lab ${ }^{19}$ (Fitzpatrick et al. 2016). In brief, the SMASH images are first reduced with the NOAO Community Pipeline (CP; Valdes et al. 2014) and then PSF photometry catalogs are generated with the DAOPHOT-based (Stetson 1987) PHOTRED pipeline. ${ }^{20}$ These catalogs are calibrated using photometric transformation equations derived from standard star fields. We refer the readers to Nidever et al. (2017) for the detailed description of the data reduction and photometry.

The photometric precision of the final SMASH catalogs is roughly $1.0 \%(u), 0.7 \%(g), 0.5 \%(r), 0.8 \%(i)$, and $0.5 \%(z)$. The obtained calibration accuracies are $1.3 \%(u), 1.3 \%(g), 1.0 \%(r)$, $1.2 \%(i)$, and $1.3 \%(z)$. The median $5 \sigma$ point source depths in the ugriz bands are $(23.9,24.8,24.5,24.2,23.5) \mathrm{mag}$, respectively, which is $\sim 2$ mag deeper than SDSS (York et al. 2000) and $\sim 1.4$ mag deeper than Pan-STARRS1 (Chambers et al. 2016). The astrometric precision is $\sim 15$ mas, and the accuracy is $\sim 2$ mas with respect to the Gaia DR1 astrometric reference frame (Gaia Collaboration et al. 2016).

Out of the 197 SMASH survey fields, we use 62 observing fields ${ }^{21}$ that cover the LMC main body (up to $\sim 5^{\circ}$ to the north and up to $\sim 10^{\circ} .5$ to the south from the LMC center) and have noticeable LMC RC populations. Our data cover the outer portion of the southern LMC disk, which is a region that has not yet been well studied. Forty of the outer LMC fields (all beyond a radius of 4.5 ) do not have the deeper exposures and

\footnotetext{
${ }^{19}$ http://datalab.noao.edu/smash/smash.php

${ }^{20}$ https://github.com/dnidever/PHOTRED

${ }^{21}$ We note that only two (Field 44 and Field 55) of these 62 fields were included in the first data release.
} 


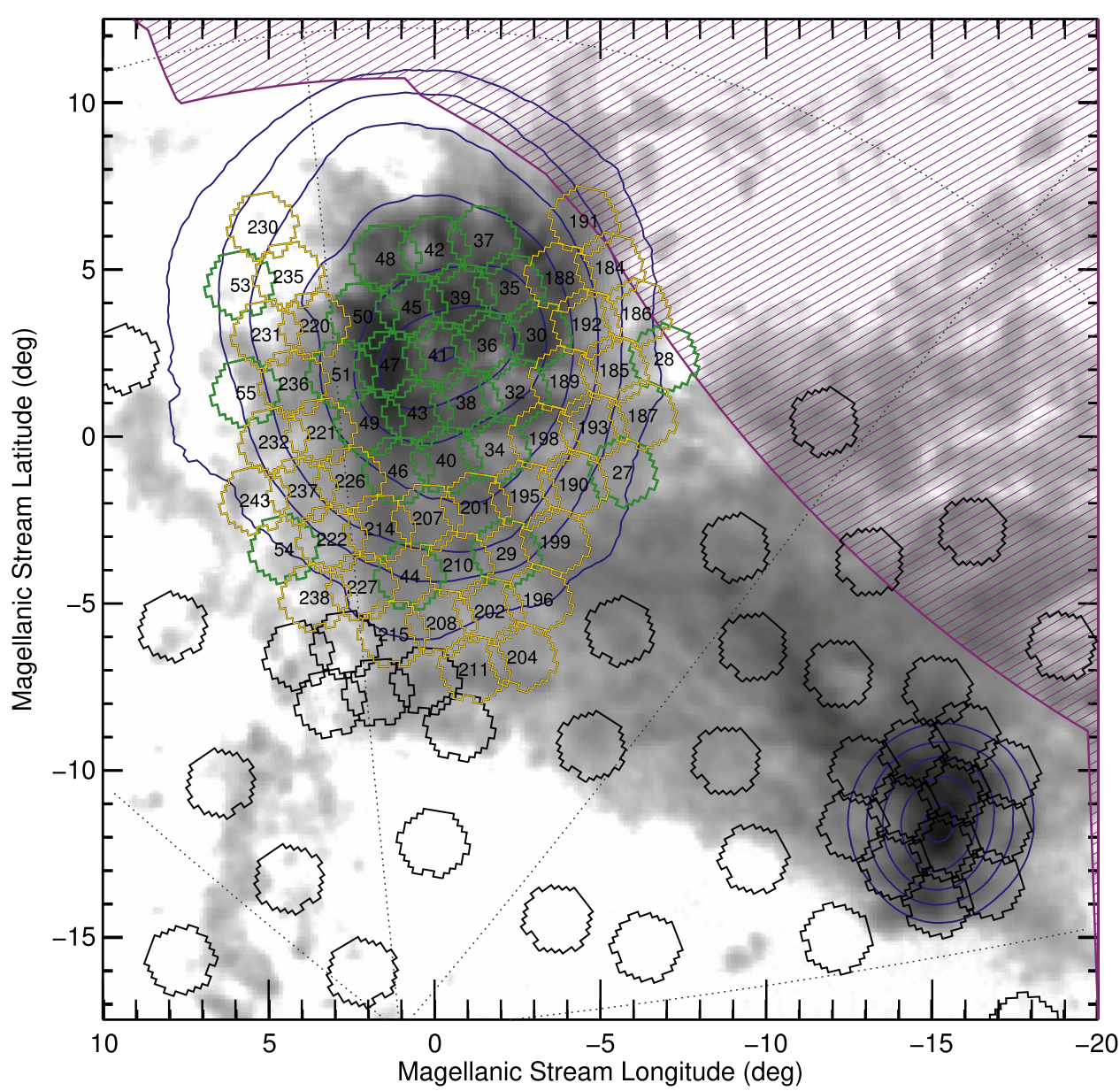

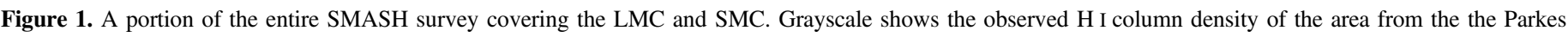

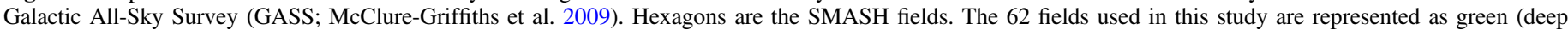

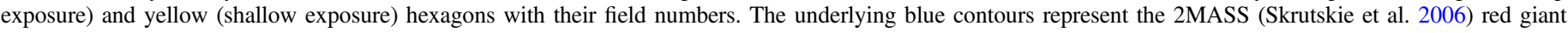
branch star counts. The purple shaded region represents the Dark Energy Survey footprint.

$u$-band data, and are therefore roughly $\sim 1.5$ mag shallower than our standard SMASH fields. However, our artificial star tests (ASTs) show that the data are still sufficiently deep for studying the LMC RC stars.

To characterize the photometric completeness of our data, we perform ASTs. Initial ASTs are run on all the SMASH fields using a single CCD. Roughly 10,000 artificial stars are added to all of the exposures for a given field. The artificial stars uniformly sample the $(g-i, g)$ CMD space $(-1.0<g-i<3.5$ and $17.0<g<27.0)$ and the spatial region on the sky covered by the images. For each image, the $(\alpha, \delta)$ coordinates are converted to pixel positions using the WCS, and the calibrated magnitudes are converted into instrumental magnitudes using the photometric transformation equations for that field. The artificial stars are then added to the image using DAOPHOT's ADDSTAR routine and the PSF of that image. Next, the images are processed with PHOTRED using the original settings and the new option for artificial stars, which skips the PSF generation and certain other steps. To determine the recovered artificial stars, the final catalog of objects are crossmatched with both the original science catalog and the input list of artificial stars. When a recovered object matched both an artificial star and an original object in position, the multiband photometry is used to find the best match.

Completeness maps in $(g-i, g)$ are generated for each field using the recovered artificial stars. The $50 \%$ completeness at the RC color of $g-i=0.8$ for LMC fields beyond a radius of $\sim 5^{\circ}$ is $g \sim 25 \mathrm{mag}$ and decreases to $g \sim 22.5 \mathrm{mag}$ in the innermost and most crowded LMC fields, but this is still three magnitudes deeper than the RC at $g=19.3 \mathrm{mag}$. The completeness improves for redder colors; therefore, the photometry for reddened and fainter RC stars is also of good quality. The shallow LMC fields are $\sim 1.5$ mag shallower than the normal SMASH fields, but the 50\% completeness for the $\mathrm{RC}$ color of $g \sim 23.5 \mathrm{mag}$ is still over four magnitudes fainter than the RC. More complete ASTs using multiple mocks and covering all of the chips for a given field are currently in progress.

Figure 2 presents example CMDs of deep and shallow exposure fields (Fields 55 and 235, respectively). Both CMDs show many detailed features associated with different stellar evolutionary stages. An MS covering many ages extends up to $i \sim 16 \mathrm{mag}$. Two subgiant branches are visible, one at an intermediate age ( $\sim 3 \mathrm{Gyr})$ and a second at a much older age $(\sim 10 \mathrm{Gyr}$ with $Z=0.004)$. There is also a prominent red giant branch (RGB), asymptotic giant branch (AGB) bump, main $\mathrm{RC}$, secondary RC (SRC; vertical feature fainter than the main $\mathrm{RC}$ ), vertical structure or blue loop (VS; vertical feature brighter than the main RC), and a horizontal branch (HB). Both the SRC and VS are in the evolutionary stage of core $\mathrm{He}$ burning, but born with a slightly higher initial mass than the main RC (Girardi 2016). The HB is the metal-poor equivalent of the RC, thus appearing on the bluer side of the main RC. The 

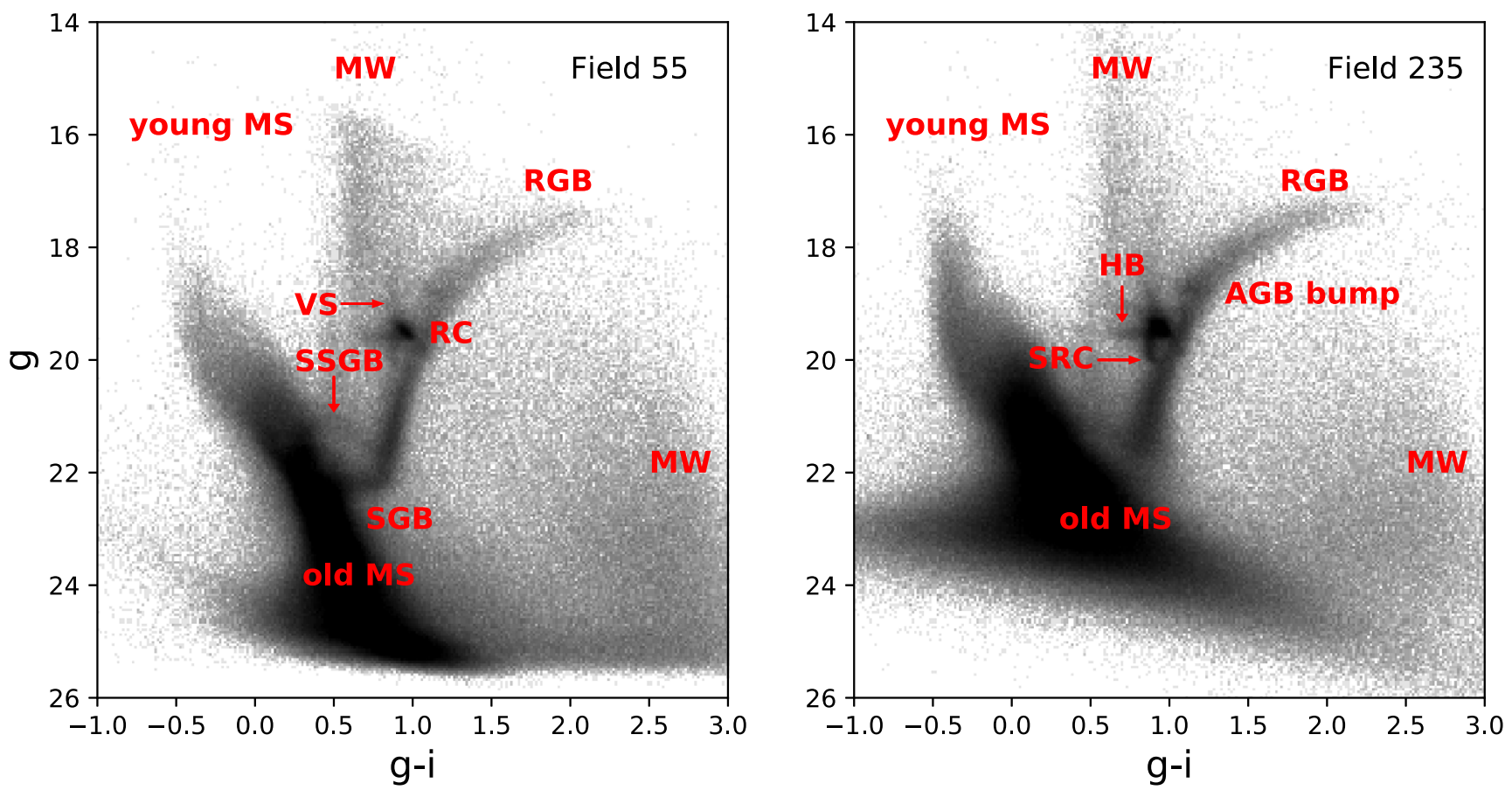

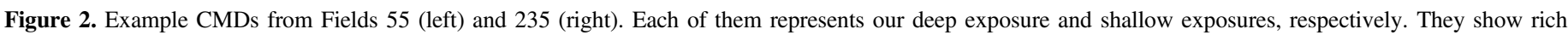

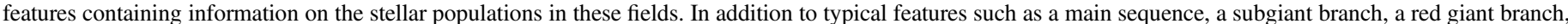

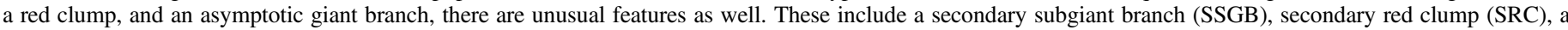

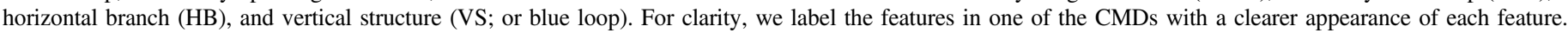

LMC RGB appears as a single broad band unlike in M31 and the SMC where the RGB is well split into visibly distinct sequences (Dalcanton et al. 2015; Yanchulova Merica-Jones et al. 2017). A single RGB sequence suggests that stars and dust in the LMC are well mixed rather than having a thin dust layer within a stellar disk. Finally, there are foreground MW stars appearing as a fairly uniform "sheet" in the CMD with a pileup of stars between the LMC MS and RGB resulting from the blue edge of the MW MS turnoff stars.

\section{RC Stars in the LMC}

The $\mathrm{RC}$ is one of the most prominent features in the CMD. In principle, the intrinsic color and brightness of the $\mathrm{RC}$ are confined to a narrow region in the CMD. Thus, the amount of dust extinction can be inferred from a shift in its observed color from the intrinsic color. Moreover, extinction-corrected magnitudes can then be used to determine the distances to individual RC stars, enabling the construction of a 3D map of a galaxy. In reality, however, the colors and magnitudes are affected by variations in the stellar populations (such as age and metallicity) due to spatial variation in star formation histories (SFHs) and metal enrichment history across the galaxy. In this study, we use the RC as extinction and distance probes of the LMC disk, with careful attention to effects other than extinction and distance that may influence our measurements. We will fully discuss the effect of variations in stellar populations in Section 7.

\subsection{Selection of RC Stars}

In each SMASH field catalog, we first select point-like sources using the DAOPHOT "chi" and "sharp" parameters: chi $<3$ ( $<5$ for Fields 36 and 41 in the center) and $\mid$ sharp $\mid<1$ $(<2$ for Fields 36 and 41). Inside the LMC main body, contamination by background galaxies is negligible, especially within the magnitude range of interest in this study.

Figure 3 describes our RC selection in each SMASH field. Selecting the RC stars based on their color and magnitude is straightforward. First, we define a large initial box (red dashed line) around the RC along the reddening vector and use it as a guiding boundary. The maximum color $(g-i=2)$ is set in order to limit the contamination by MW foreground stars. To further minimize the contaminants in the RC selection, we carefully adjust the initial RC selection box by eye for each field, excluding stellar populations other than the main RC. The determined color range varies for each field mainly according to their relative dust extinction, while the slope is almost parallel to the reddening vector. Traditionally, other studies have used simple square selection boxes around the RC. For our CMDs, we found that such an approach led to an unacceptably high contamination of RGB stars and did not capture the entire $\mathrm{RC}$ in areas with high internal differential extinction. Once we select RC stars in each observing field, we remove duplicates in the overlapping regions among fields. The number of unique RC stars in our final catalog is $\sim 2.2$ million.

Figure 4 shows the number count map of the selected RC stars at $10^{\prime}(150 \mathrm{pc})$ spatial resolution. We keep the same $10^{\prime} \times 10^{\prime}$ spatial cell size for all subsequent analysis. We also conduct the same analysis at a much higher resolution of $40^{\prime \prime}$ $(10 \mathrm{pc})$ and conclude that our main results are robust against the choice of spatial resolution. In general, the RC stars show an extended spatial distribution with smoothly decreasing number density with galactic radius, compared to the more concentrated and patchy distribution of young stars (e.g., Zaritsky et al. 2004)— their star count map of young MS clearly shows a star-forming bar and one-armed spiral pattern. We note that high dust extinction makes our RC selection slightly incomplete only in the very central region $(\rho<0$.5). In the 
(a)
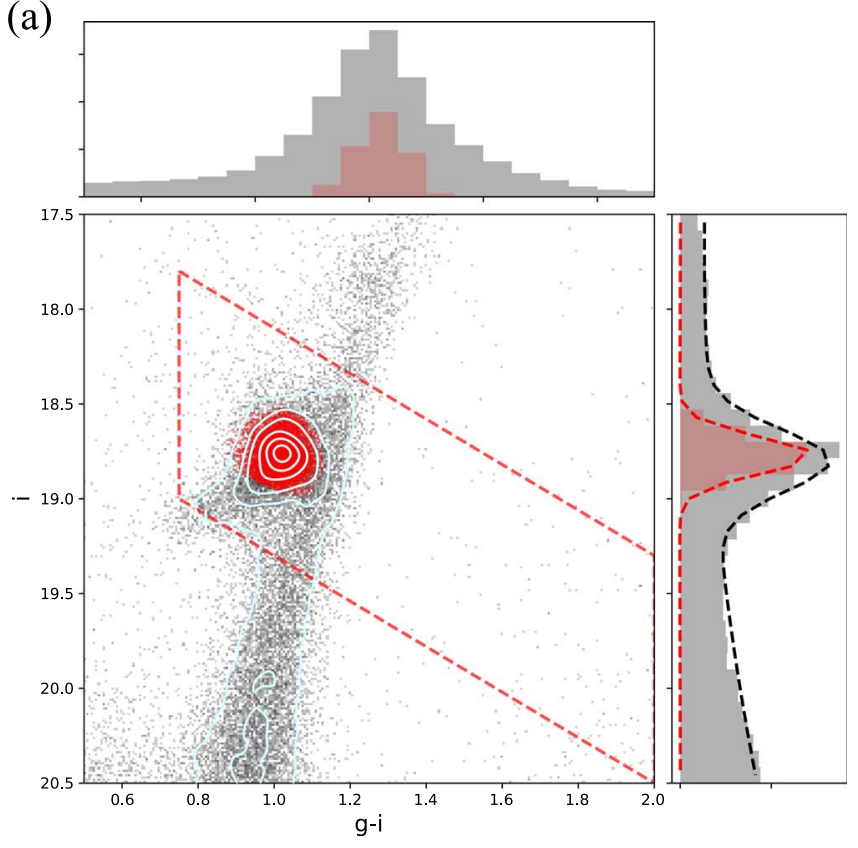

(b)
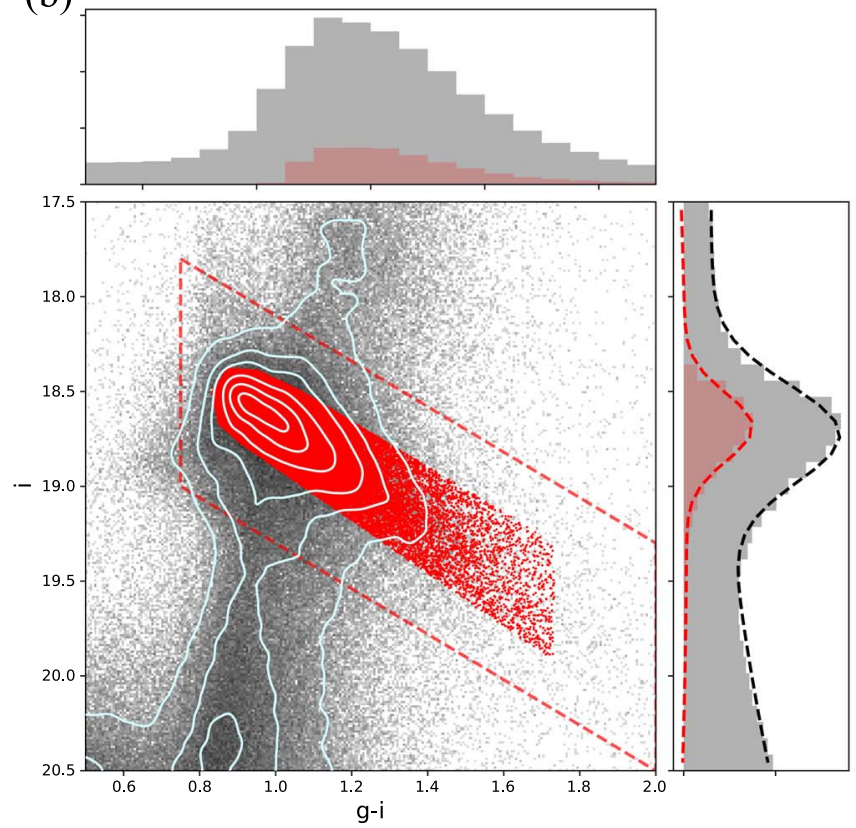

Figure 3. Zoomed CMDs showing selected RC stars using our polygon (red dots) for Field 190 (top) as an example of a low-extinction field and Field 50 (bottom) as an example of a high-extinction field. The red dashed line shows an initial boundary to guide our selection. The slope roughly follows the reddening vector for $R_{V}=3.4$, which is an average $R_{V}$ for the LMC (Gordon et al. 2003). Our RC selection is conservative in that the selection minimizes contaminants of RGB, HB, SRC, and VS stars. For each CMD, we also present marginal histograms of the color and magnitude for both all stars (gray) and the selected RC stars (red) in the top and right-hand panels, respectively. A Gaussian plus second-order polynomial fit to each magnitude distribution is shown as a dashed line.

rest of the disk with low crowding and low extinction, our RC selection is almost $100 \%$ complete. The observed number count map itself already shows some interesting structures. We investigate the LMC disk structure for various stellar populations, such as RC and young MS, by modeling their observed

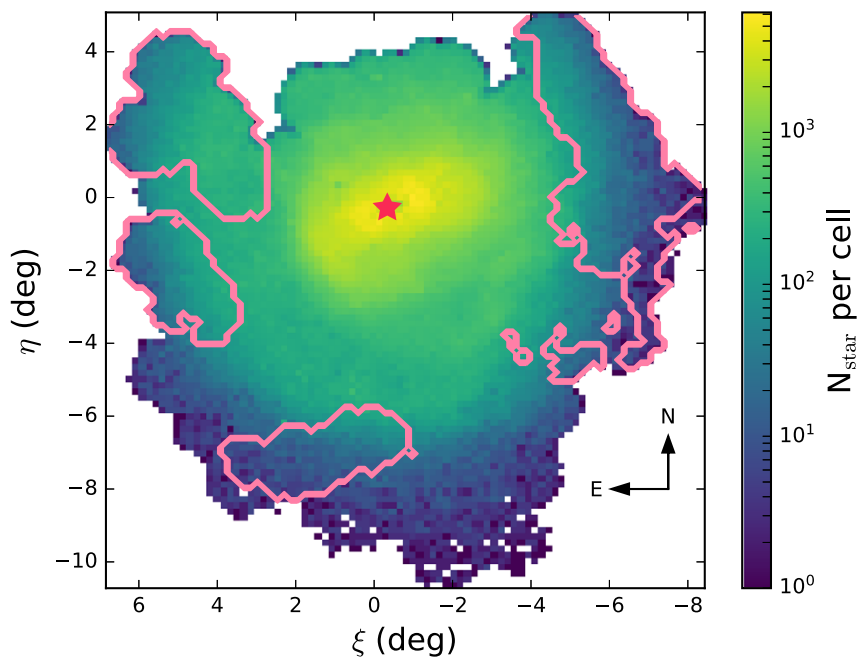

Figure 4. RC star count map in stars per $10^{\prime} \times 10^{\prime}$ pixel. North is up and east is left. The red star indicates the center of the bar (van der Marel \& Cioni 2001). The pink line outlines the region where the "clean" RC sample (i.e., RCs with zero or a negligible amount of internal dust) is distributed over (see Section 4.1). The electronic version of the RC count map is available as supplementary material to this paper. The data used to create this figure are available.

star count map as a 2D projection of a tilted elliptical disk model in a separate paper (Choi et al. 2018).

\section{2. $R G B$ Contamination}

Because the RGB usually overlaps with the RC in an observed optical CMD, it is impossible to completely remove RGB contaminants from the RC sample without extra information from spectroscopic data on surface gravity and effective temperature (e.g., Bovy et al. 2014; Nidever et al. 2014). Infrared (IR) color information could be helpful to distinguish the RC from RGB to some degree (e.g., Majewski et al. 2011). However, there are no publicly available IR survey data comparable with our optical data in terms of depth, photometric precision, and areal coverage even for the RC brightness level.

Fortunately, measuring extinction and distance does not depend significantly on the small fraction of RGB contaminants because they might induce only a small bias in the $\mathrm{RC}$ color and magnitude due to their similar color and magnitude compared to those of the RC, especially in each small $10^{\prime}$ cell. Nevertheless, we estimate the fraction of possible RGB contaminants in our RC sample in each SMASH field.

In Figure 3, we also show histograms of the color and magnitude for a given CMD. A Gaussian profile can describe the main RC distribution, and a second-order polynomial can describe the underlying distribution of contaminants including RGB stars (e.g., Stanek \& Garnavich 1998; Olsen \& Salyk 2002). We fit the magnitude distribution of stars near the RC with a Gaussian profile combined with a second-order polynomial and find that the bright and faint end magnitudes of our selected RC stars are mostly within $2 \sigma$ from the Gaussian mean, except for highextinction fields. We calculate the RGB contamination fraction within our RC selection polygon based on the second-order polynomial terms. On average, the RGB contamination fraction in our sample is $\sim 10 \%$. If one uses a simple square box for the RC selection, the contamination fraction can be as high as 

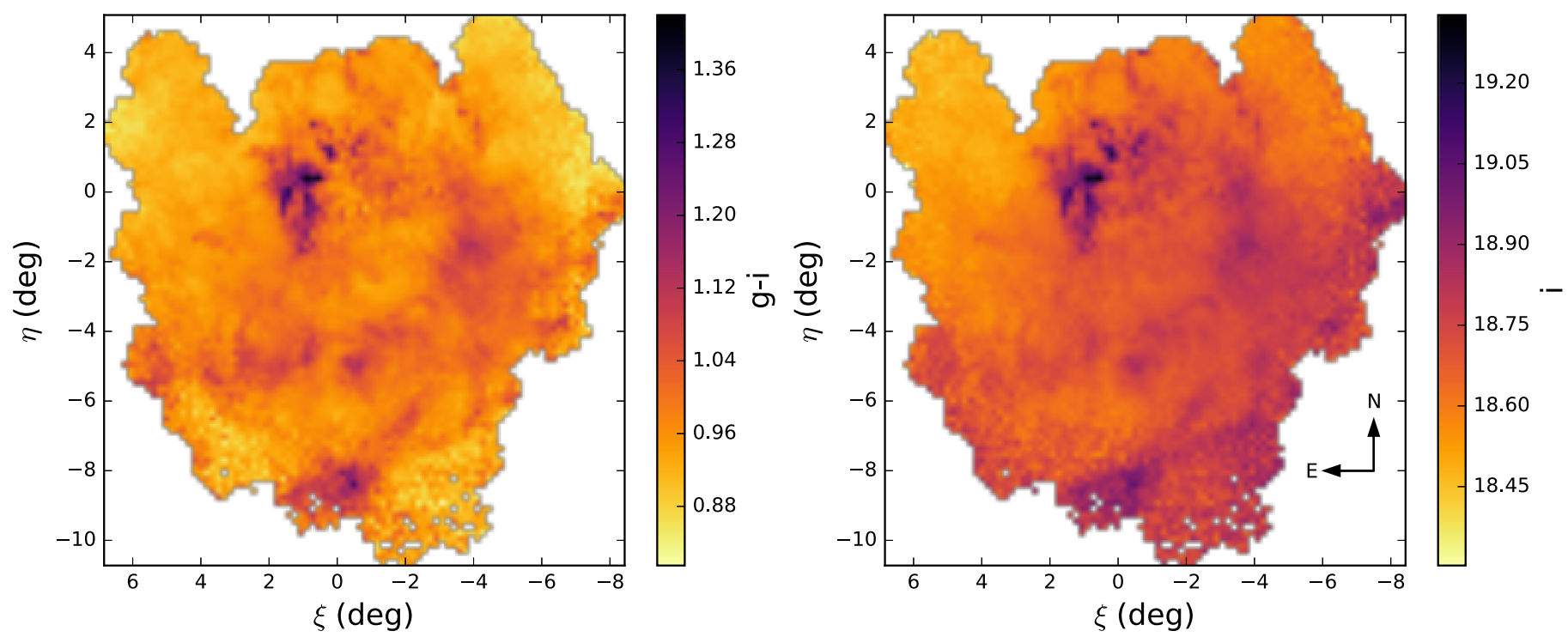

Figure 5. Maps of the median $g-i$ color (left) and $i$-band magnitude (right) of the RC stars. The observed color map itself already traces well the dust emission in the inner disk as well as the MW galactic cirrus in the outer disk. The observed magnitude map shows an additional spatial gradient; the RC magnitude increases from northeast to southwest. These maps are available as supplementary material to this paper. The data used to create this figure are available.

$\sim 30 \%-40 \%$ by including additional populations on the bluer side (i.e., HB, SRC, and VS) as well as more RGB stars. This high contamination fraction would result in significant systematic errors in extinction and distance measurement by shifting the average color and magnitude from those of true RC stars.

\subsection{Observed Magnitude and Color Maps}

We first produce maps of the median observed color and magnitude at each $10^{\prime}$ cell, from which we will derive the reddening and distance. While the mean $\mathrm{RC}$ color and magnitude have typically been calculated by fitting the distributions with a Gaussian profile combined with a second-order polynomial, we take the median magnitude and color for each cell. Due to our conservative RC selection, we found no significant contribution from second-order polynomial terms when describing the selected RC's magnitude distribution in each $10^{\prime}$ cell. Thus, there is no significant difference between the geometric median and the Gaussian mean, except for cells at the edge of the maps that suffer from small number statistics where the geometric median provides more robust measurements.

Figure 5 presents the maps of observed (i.e., undereddened) median $g-i$ color and $i$-band magnitude, which is less affected by dust than the bluer DECam filters and more complete than the $z$-band. The observed color map traces the dust emission in the inner disk (see Figure 6 in Gordon et al. 2014) as well as the MW galactic cirrus in the outer disk (Schlegel et al. 1998). In addition, the observed magnitude map shows a spatial gradient with magnitude increasing from the northeast to the southwest. The LMC disk is known to be tilted in a way such that the northeast is closer (brighter) to us and the southwest is farther away (fainter) from us (e.g., Caldwell \& Coulson 1986; van der Marel \& Cioni 2001; Olsen \& Salyk 2002; Mackey et al. 2016). We measure the inclination (i) and line-of-nodes position angle $(\theta)$ of the LMC disk using the RC in Section 6.

\section{Intrinsic Properties of the RC}

For a given intrinsic color and magnitude of the $\mathrm{RC}$, the observed color provides information about the line-of-sight dust (i.e., from reddening), while the observed magnitude is altered both by the dust and the RC distance. Although the intrinsic brightness and color of the $\mathrm{RC}$ are a function of stellar age and metallicity (i.e., stellar population), only a mild population gradient is expected in the LMC disk. This is because the LMC disk shares relatively uniform SFHs for intermediate/old stellar populations (e.g., Harris \& Zaritsky 2009; Weisz et al. 2013; Monteagudo et al. 2018). The metallicity gradient is also known to be mild across the LMC disk (e.g., Feast et al. 2010). Thus, one can expect no severe effects from stellar population differences across the LMC on the RC colors and magnitudes, except for the central region where the average age of the stellar population is younger.

Although the LMC's population gradient is not expected to be severe, because we aim for a high-quality reddening map and an accurate distance measurement of the LMC disk, it is necessary to quantify the spatial variations in the intrinsic color and magnitude of the RC from differences in stellar populations in order to minimize and quantify systematic uncertainties in our 3D structure measurements of the LMC disk.

\subsection{Intrinsic Color of the Red Clump}

\subsubsection{Derived Intrinsic RC Color from a "Clean" RC Subsample}

To derive the intrinsic color distribution of the $\mathrm{RC}$, we first construct a subsample of the $\mathrm{RC}$ in the regions with no or negligible internal dust. Because dust in front of these RC stars comes solely from the MW foreground, which acts as a dust screen, it is easy to break the degeneracy between the internal line-of-sight dust and the effect of stellar populations on their observed color. Using those RC stars enables us to derive their intrinsic colors after correcting for MW foreground dust.

To construct the RC subsample, we start by selecting 18 SMASH fields that do not show noticeable reddened RC 

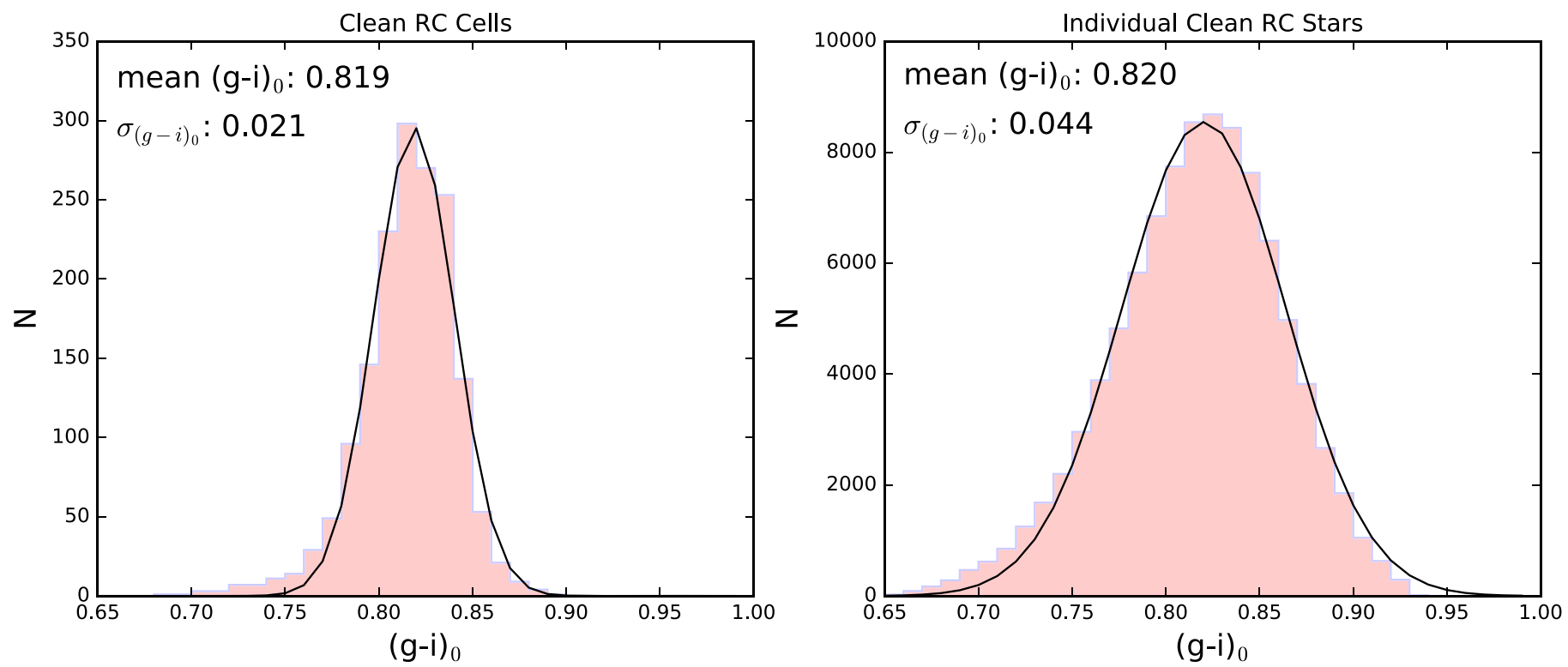

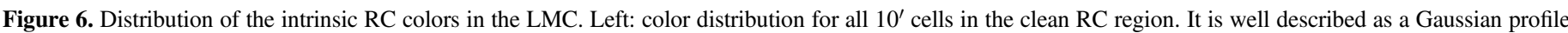

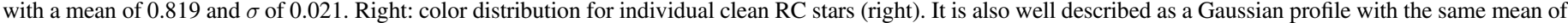

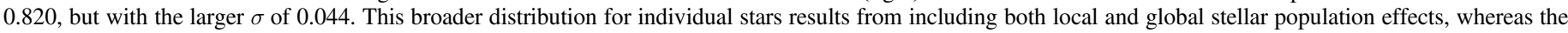
distribution for all cells reflects the global population effect only.

"streaks" in their CMDs after correcting for MW foreground dust $^{22}$ indicating zero or a negligible amount of internal dust. It is highly unlikely that all RC stars in one SMASH field (a field of view of $\sim 3 \mathrm{deg}^{2}$ ) experience the same amount of internal dust, and thus do not develop a streak. For the MW dust correction in these 18 observing fields, we use the Schlegel et al. (1998, hereafter SFD98) reddening map, which was derived from infrared dust emission. To convert SFD98 $E(B-V)$ to $E(g-i)$, we use updated reddening coefficients $^{23}$ with $R_{V}=3.1$ for the DECam standard bandpasses that reflect Schlafly \& Finkbeiner's (2011) calibration adjustment (Abbott et al. 2018). Although the above 18 fields are located outside the regions where the emission from the LMC dominates FIR emission, a tiny fraction of 3 fields out of 18 overlaps with the inner region of the LMC where the internal dust becomes dominant. We first exclude RC stars residing in these overlapping regions. From the remaining RC stars in the 18 observing fields, we further exclude stars with SFD98 $E(B-V)$ values larger than 0.099 (outside $1 \sigma$ of the mean) to minimize potential contamination by $\mathrm{RC}$ stars that might be slightly reddened by a small amount of internal dust without developing a clear streak feature in a CMD. We designate this internal-dust-free RC sample as the "clean" RC sample, which accounts for $\sim 4 \%$ of our RC star catalog. The clean RC sample is distributed over the regions that are outlined in pink in Figure 4 . The median SFD98 $E(B-V)$ value of the clean RC sample is $0.065 \mathrm{mag}$, which is consistent with a typical reddening value of $0.075 \mathrm{mag}$ within uncertainty toward the LMC that was estimated based on the median dust emission arising from surrounding annuli (SFD98).

Using only the clean RC sample, we compute the median intrinsic color for each $10^{\prime}$ cell over the clean $\mathrm{RC}$ region outlined by the pink line in Figure 4. In Figure 6, we present

\footnotetext{
22 These fields are Fields 27, 28, 44, 53, 55, 184, 185, 186, 187, 190, 191, 193 , $220,227,230,232,235$, and 238.

${ }^{23}$ Reddening coefficients for other $R_{V}$ values are kindly provided by $\mathrm{K}$. Bechtol (2017, private communication).
}

their median intrinsic color distribution (left) as well as the intrinsic color distribution of individual clean RC stars (right) for completeness. Both distributions are well described as a Gaussian profile with an identical mean $(g-i)_{0}$ of $\sim 0.82$, but with different widths, with narrower width for the cells' median color distribution, as expected. The median RC color distribution of $10^{\prime}$ cells reflects the cell-to-cell variation in SFH and chemical enrichment (i.e., global variations in stellar populations). The color distribution of individual clean RC stars, on the other hand, reflects both the full SFH and chemical enrichment history sampled in each cell (i.e., the local distribution of stellar populations) and their spatial variation. Almost all of the $10^{\prime}$ cells exhibit standard deviations larger than $\sim 0.02$ in their intrinsic color distribution of individual clean RC stars. The fact that the median color distribution of $10^{\prime}$ cells in the clean $\mathrm{RC}$ region is narrower than the color distribution of individual clean RC stars per cell suggests that global variations in stellar populations across the LMC are moderate, at least for those populations that produce RC stars.

\subsubsection{Intrinsic Color Radial Profile}

In Figure 7, we investigate a radial profile of the intrinsic color using the clean RC sample. Because the clean RC sample consists of stars selected in the restricted area (see Figure 4), it covers a limited range of galactic radius ( $\rho$ in degrees) from $\sim 2.7$ to $\sim 8^{\circ} .5$. The grayscale shows the distribution of the intrinsic colors of individual cells as a function of galactic radius from the LMC center, and the blue solid line presents a mean color with an associated error in each 0.2 width radial bin. The red shaded line shows the best fit to the gray data characterizing the overall radial profile of the observed intrinsic RC color. We find no statistically meaningful radial dependence of the intrinsic $\mathrm{RC}$ color between $4^{\circ}$ and $7^{\circ}$. In this radial range, there is basically no change in the mean color with galactic radius, but there is a larger scatter around the median colors at larger radius. On the other hand, the intrinsic RC color tends to be slightly bluer both toward the 


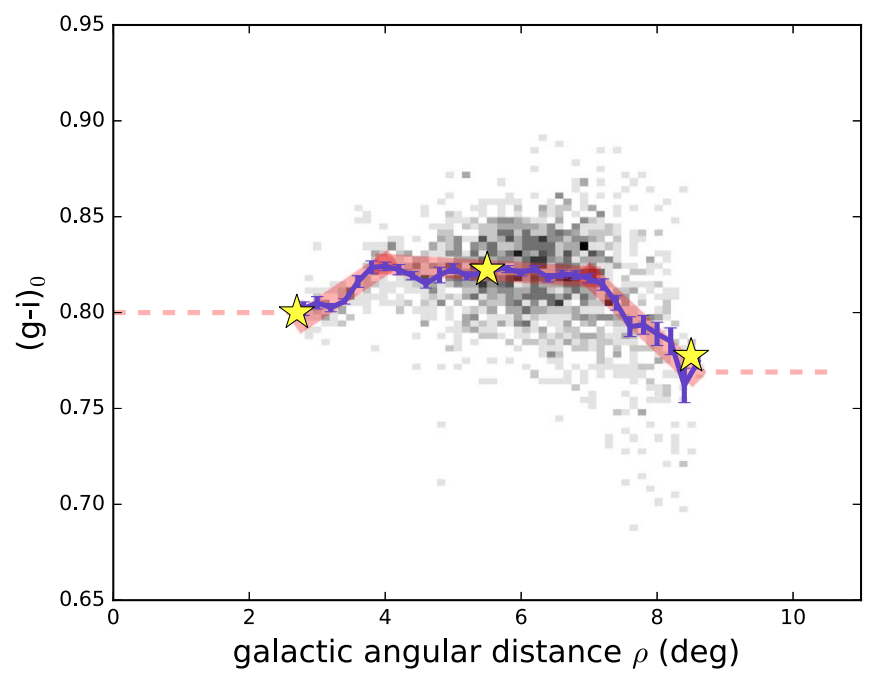

Figure 7. Radial dependence of the intrinsic RC color constructed from the "clean" (i.e., zero or negligible internal dust) RC sample. The $\rho$ denotes galactic distance from the LMC center in degrees. The blue line shows the mean intrinsic color with an error bar in each 0.2 radial bin between $2.7<\rho<8$. 5 . This limited radial range is due to the restricted radial coverage of the clean RC sample. The red shaded line is the best fit to the underlying grayscale data, and it is our default radial profile for the intrinsic RC color between $2.7<\rho<8.5$. We measure an almost zero slope between $4^{\circ}<\rho<7^{\circ}$ with a constant color of 0.822 , a slope of $0.024 \mathrm{dex} \mathrm{deg}^{-1}$ between $2.7<\rho<4^{\circ}$, and a slope of $-0.033 \mathrm{dex} \mathrm{deg}^{-1}$ between $7^{\circ}<\rho<8.5$. The red dashed line shows our default radial profile for the intrinsic RC color at $\rho<2.7$ and $\rho>8.5$. The yellow stars present the predicted $g-i$ color from the known metallicity gradient and the agemetallicity relation for the LMC disk at $\rho=2.7,5.5$, and 8.5 .

center, with a slope of $0.024 \mathrm{dex} \mathrm{deg}^{-1}$, and the outer galaxy, with a slope of $-0.033 \mathrm{dex} \mathrm{deg}^{-1}$.

This RC color radial trend can be interpreted as a result of more metal-rich, but younger, populations in the inner region and older, but more metal-poor, populations in the outer region. An outside-in formation has been suggested for the LMC, supported by the observational evidence that old and metalpoor stars are preferentially found in the outer disk, whereas young and metal-rich stars are found in the inner disk (e.g., Gallart et al. 2008; Meschin et al. 2014). Although deriving full SFHs can provide a complete picture for the intrinsic RC properties across the LMC disk, this is beyond the scope of this paper. In a future paper, we will map the full SFHs using the SMASH data (C. Gallart et al. 2018, in preparation).

To verify whether the detected radial color trend is physically reasonable, we conduct a simple comparison of the intrinsic RC colors with PARSEC isochrones (Bressan et al. 2012; Marigo et al. 2017) by using previously reported metallicity gradients and age-metallicity relations (AMRs) in the LMC disk (Carrera et al. 2008, 2011; Piatti \& Geisler 2013; Choudhury et al. 2016; Pieres et al. 2016) as a guide. These previous studies reported a shallow metallicity gradient with the mean $[\mathrm{Fe} / \mathrm{H}]$ decreasing from -0.4 in the star-forming bar region to -0.8 to $\sim-1.0$ in the outer region $\left(\geqslant 7^{\circ}\right)$. There is no solid metallicity measurement for stars in the southern regions of the LMC outer disk yet. However, our CMDs can provide a loose constraint on it. For example, lowmetallicity populations develop an extended horizontal branch feature in the CMD. We found no prominent extended $\mathrm{HB}$ features in any of our CMDs across the LMC disk, indicating that the minimum metallicity of the dominant stellar populations cannot be lower than $[\mathrm{Fe} / \mathrm{H}] \simeq-2$ even in the outer disk. Therefore, we set the minimum average $[\mathrm{Fe} / \mathrm{H}]$ as -1.0 , which is the minimum average $[\mathrm{Fe} / \mathrm{H}]$ in the northern regions of the the LMC outer disk (Carrera et al. 2008).

We first compute the mean absolute $g$ - and $i$-band magnitude as well as the mean $g-i$ color only for core He-burning stars from an isochrone at a given age and metallicity by following Girardi \& Salaris (2001; see their Equations (3) and (4)). The age is in steps of $\Delta \log ($ age $)=0.5$, and the metallicity ranges from $[\mathrm{Fe} / \mathrm{H}]=-0.4$ to -1.0 in steps of $0.1 \mathrm{dex}$. We use the Chabrier initial mass function to be consistent with the PARSEC isochrones used in this study. The calculated model $\mathrm{RC}$ colors and magnitudes show the same behavior presented in Girardi \& Salaris (2001) and Girardi (2016). In short, the RC stars older than $\sim 2$ Gyr behave in a simple way-they become fainter and slightly bluer (redder) as the age (metallicity) increases. However, the colors and magnitudes for RC stars younger than $\sim 1.5 \mathrm{Gyr}$ vary rapidly with age and behave differently for different metallicities. We refer the readers to Girardi \& Salaris (2001) and Girardi (2016) for the detailed discussion about population effects on the RC photometric properties.

We compare these calculated model mean colors for a given age and metallicity with the clean RC sample's intrinsic colors in each radial range: (i) $2.7-4^{\circ}$, (ii) $4^{\circ}-7^{\circ}$, and (iii) $7^{\circ}-8^{\circ} .5$. For these three radial bins, we adopt representative $[\mathrm{Fe} / \mathrm{H}]$ values of $-0.5,-0.7$, and -0.8 , respectively, which reflect the reported metallicity gradient in the LMC (Carrera et al. 2008, 2011; Piatti $\&$ Geisler 2013; Pieres et al. 2016). Combining these three representative metallicities with the known AMR (Carrera et al. 2008; Piatti \& Geisler 2013), we derive stellar ages of $\sim 1.6 \mathrm{Gyr}, \sim 5.6 \mathrm{Gyr}$, and $\sim 6.3 \mathrm{Gyr}$ for these radial bins by finding models that best match our observed intrinsic colors. The best model colors in each radial bin are marked as yellow stars in Figure 7.

For the innermost radial bin $\left(0^{\circ}-2^{\circ} .7\right)$, we adopt the intrinsic RC color at $\rho=2.7$ as a default color, and for the outermost radial bin ( $\left.8.5-10^{\circ} .5\right)$, we adopt the intrinsic $\mathrm{RC}$ color at $\rho=8.5$ as a default color rather than introducing additional color gradients in these radial bins.

We, however, can attempt to infer physically reasonable intrinsic RC colors both inside $\rho=2^{\circ} .7$ and outside $\rho=8.5$. For example, we can rule out the possibility of extrapolating the inner color gradient $\left(\sim 0.024 \mathrm{dex} \mathrm{deg}^{-1}\right)$ to the innermost radial bin. This is because the metallicity gradient in the central bar region has been known to be shallow with a mean $[\mathrm{Fe} / \mathrm{H}]$ of about -0.4 dex (e.g., Carrera et al. 2008, 2011; Choudhury et al. 2016). If we extrapolate the inner color gradient of $\sim 0.024 \mathrm{dex} \mathrm{deg}^{-1}$ to the very center of the LMC, this predicts a $g-i$ color of $\sim 0.73$. However, none of the metal-rich $([\mathrm{Fe} / \mathrm{H}]>-0.5)$ isochrones have colors that blue and, in fact, all of the extrapolated colors for $\rho<2.7$ are too blue (which are bluer than $g-i \simeq 0.8$ ) for the metal-rich isochrones, even with very young ages. Therefore, this color extrapolation to smaller radii does not appear physically reasonable.

In Section 7.2, we will investigate physically reasonable intrinsic RC colors in the innermost and outermost radial bins, and fully discuss the dependence of the 3D structure determination on the choice of the intrinsic RC colors.

\subsection{Intrinsic Magnitude of the Red Clump}

Girardi \& Salaris (2001) measured the effect of stellar population variations in the mean $\mathrm{RC}$ magnitude for some fields across the LMC disk based on spatially resolved SFHs 
and AMRs, and reported the maximum difference of $0.036 \mathrm{mag}$ in the $I$-band magnitude among different regions. Using the clean $\mathrm{RC}$ sample, we also derive the intrinsic magnitude of the RC after correction for both MW dust and its gradual variation across the inclined LMC disk by assuming that the relative distances (due to the inclined disk) are the primary cause of the magnitude differences observed among the cells (van der Marel \& Cioni 2001).

First, we compute the median $i$-band magnitude for all $10^{\prime}$ cells in the clean $\mathrm{RC}$ region after correcting for the extinction by the foreground MW dust. We then adopt the median MW extinction-corrected $i$-band magnitude as a "fiducial" RC magnitude to obtain the relative distances to each other. These relative distances are converted to the absolute distances by putting the LMC center at $D_{0}=49.9 \mathrm{kpc}$ (de Grijs et al. 2014). We use the absolute distances $(D)$ and celestial coordinates (right ascension $\alpha$, declination $\delta$ ) of each cell to compute their Cartesian coordinates $(X, Y, Z)$.

To perform the coordinate transformation from the celestial coordinates and distance to Cartesian coordinates, we adopt the LMC disk's photometric center, $\left(\alpha_{0}, \delta_{0}\right)=\left(82^{\circ} .25,-69^{\circ} .5\right)$, as the origin (van der Marel 2001; van der Marel \& Kallivayalil 2014).

Cartesian coordinates $(X, Y, Z)$ with the origin $\left(\alpha_{0}, \delta_{0}\right)$ are defined as follows:

$$
\begin{aligned}
& X=-D \sin \left(\alpha-\alpha_{0}\right) \cos (\delta), \\
& Y=D \sin (\delta) \cos \left(\delta_{0}\right)-D \sin \left(\delta_{0}\right) \cos \left(\alpha-\alpha_{0}\right) \cos (\delta), \\
& Z=D_{0}-D \sin (\delta) \sin \left(\delta_{0}\right)-D \cos (\delta) \cos \left(\delta_{0}\right) \cos \left(\alpha-\alpha_{0}\right),
\end{aligned}
$$

where the $X$-axis is antiparallel to the right ascension axis, the $Y$-axis is parallel to the declination axis, and the $Z$-axis is toward the observer.

We fit a plane to the $(X, Y, Z)$ positions of all clean $\mathrm{RC}$ cells by minimizing their distances to the plane using an optimization algorithm (scipy.optimize.leastsq). The fitting yields an inclination angle of $27^{\circ} .81 \pm 0.23$ and position angle of $146^{\circ} .37 \pm 0.37$. From this fitted plane for the clean RC sample, we compute the expected magnitude gradient across the LMC disk and correct the extinction-corrected $i$-band magnitude for this expected geometric effect. This MW dust and inclination correction yields the intrinsic RC magnitude. We note that the inclination and position angle measured based on only the clean RC sample is not our final measurement (see Section 6.1 for the final measurement), but there is no significant change in the resulting intrinsic RC magnitude with our final measurements of the inclination angle $\left(\sim 26^{\circ}\right)$ and position angle $\left(\sim 149^{\circ}\right)$. Between these two sets of the inclination and position angles, the maximum geometric effect in magnitude is found to be $\sim 0.03 \mathrm{mag}$, and the magnitude differences in most areas are comparable to our photometric uncertainty.

Because we assume that the relative distance is the primary cause of the relative magnitude difference among cells, the residuals in the inclination-corrected magnitudes might allow us to characterize the "marginal" population effects in the intrinsic RC magnitude distribution. In fact, assuming that the inclination is the primary cause is physically reasonable in terms of the expected mild stellar population gradient across the disk. Thus, the residual differences in the magnitudes after correction for first-order inclination should contain information on the effect of variation in stellar populations across the LMC on the $\mathrm{RC}$ intrinsic magnitudes.
In Figure 8, we present the intrinsic RC magnitude distributions of the cells (left) and individual stars (right). Although both distributions roughly follow a Gaussian profile with the same mean magnitude of $\sim 18.47$, the distribution of individual stars is slightly skewed to brighter magnitudes. Bright stars contributing to this skewness are confined into the end of the prominent spiral arm, suggesting these bright stars might be younger on average. Since RC brightness responds to stellar age more sensitively than color for a given metallicity, a lack of skewness in the color distribution of individual stars can be understood. Nevertheless, the width $(\sim 0.1 \mathrm{mag})$ of the individual stars' magnitude distribution is consistent with the theoretical dispersion in magnitude due to local age and metallicity spread (e.g., Girardi \& Salaris 2001; Yanchulova Merica-Jones et al. 2017). Furthermore, we find an excellent agreement between the width $(0.031 \mathrm{mag})$ of the cells' distribution, which reflects a global variation in stellar population effect across the LMC disk, and the maximum $I$-band magnitude difference of 0.036 mag that is measured among different regions in the LMC (Girardi \& Salaris 2001).

Figure 9 presents the radial profile of the intrinsic RC magnitude in the $i$-band. In contrast to the radial color dependence, the radial profile of the intrinsic RC magnitude is rather flat across the entire observed radial range, with only a slightly brighter $(\sim 0.03 \mathrm{mag})$ magnitude in the radial bin, $2.7<\rho<4^{\circ}$, which is likely due to younger populations toward the central region. This magnitude radial profile shows excellent agreement with the expected magnitudes (marked as yellow stars) from the same age, metallicity models that explain the measured intrinsic RC color radial profile (see Section 4.1.2). Thus, we adopt the mean magnitude of 18.476 as a constant intrinsic RC magnitude between $4^{\circ}<\rho<8.5$, and adopt a slope of $0.019 \mathrm{mag} \mathrm{deg}^{-1}$ between $2.7<\rho<4^{\circ}$. As with the color radial profile, we set the magnitudes at $\rho=2.7$ and 8.5 as the default values in the innermost $\left(\rho<22^{\circ} 7\right)$ and outermost $\left(\rho<8^{\circ} .5\right)$ radial bins, respectively. We will also discuss how the choice of different magnitudes affects the resulting 3D structures in Section 7.2.

\section{A Reddening Map of the LMC Disk}

Figure 10 presents the reddening map derived by RC color, showing the median reddening along the line of sight toward each cell. We compute the extinction for each cell by comparing the inferred intrinsic color from Section 4.1 and the observed median color of the $\mathrm{RC}$ at a given cell: $E(g-i)=(g-i)_{\mathrm{obs}}-(g-i)_{0}$. The average LMC reddening is $E(g-i)=0.15 \pm 0.05$, which is in good agreement with the previously reported average reddening measurements using intermediate/old stars in the LMC (e.g., Zaritsky et al. 2004; Haschke et al. 2011). The electronic version of our reddening map is available.

In the right panel of Figure 10, we also present the SFD98 reddening map derived from the dust emission. In the region outside the dashed box, our RC reddening map shows excellent agreement with the SFD98 map in both qualitative and quantitative aspects. SFD98 derived $E(B-V)$ using dust emission at $100 \mu \mathrm{m}$ assuming only $\mathrm{MW}$ dust $\left(R_{V}=3.1\right)$, and thus we also assume MW dust when converting SFD98 $E(B-V)$ to $E(g-i)$. We note for the reader that SFD98 reported that for regions containing the LMC, their reddening measurements are unreliable because of insufficient angular resolution to resolve the complex temperature structure toward 

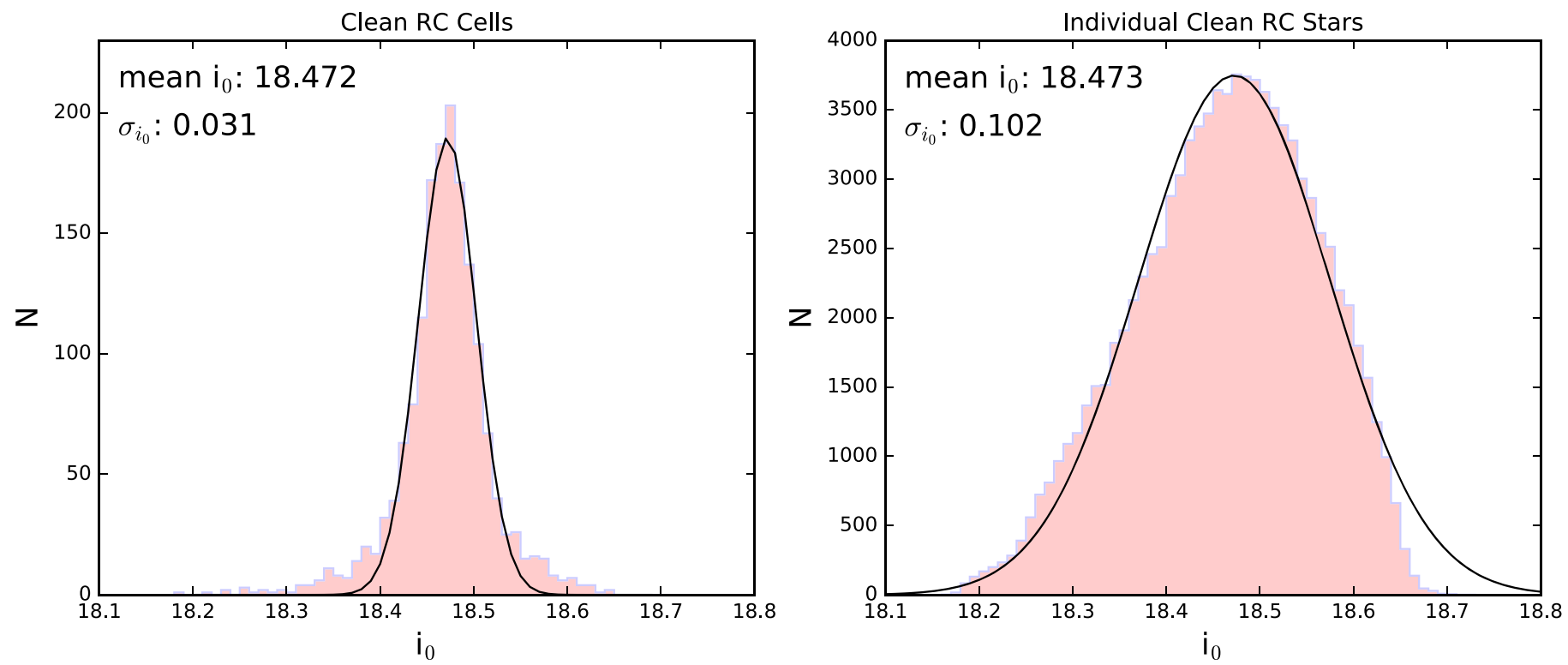

Figure 8. Same as Figure 6, but for the intrinsic $i$-band magnitude derived from the clean RC sample after correcting for dust extinction and inclination effect. The intrinsic $i$-band magnitude distribution for all $10^{\prime}$ cells is shown on the left, and that for individual clean RC stars on the right. They have the same mean $i$-band magnitude of $\sim 18.47 \mathrm{mag}$, but the distribution for individual stars is more than three times broader than that of the $10^{\prime}$ cells, again due to the contribution from both local and global population effects. The $\sigma$ of $0.031 \mathrm{mag}$ for the cells' median $i$-band magnitude distribution, which represents the global population effect, is consistent with the amount of population effect in the I-band across the LMC fields (Girardi \& Salaris 2001).

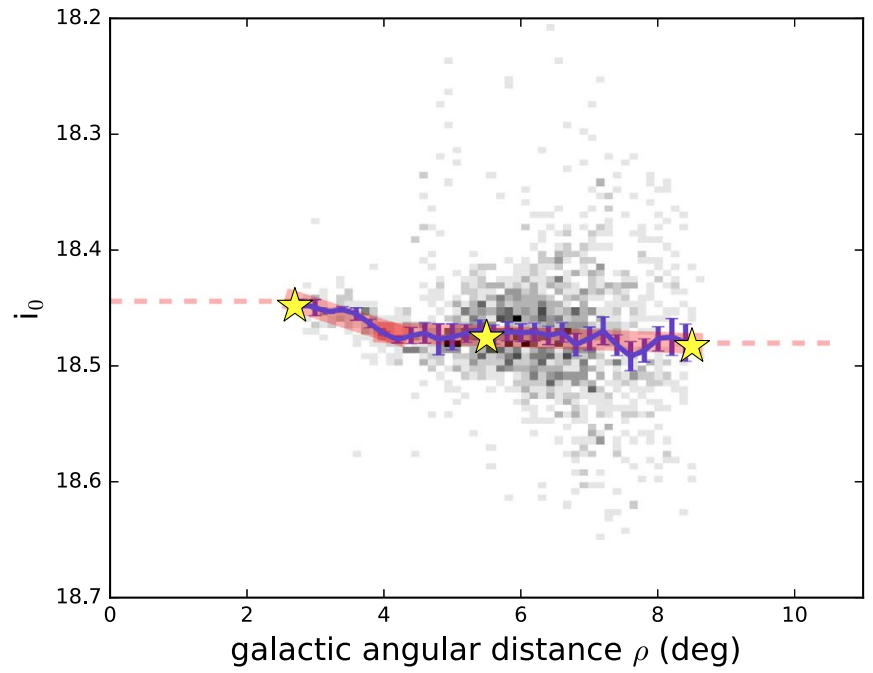

Figure 9. Same as Figure 7, but for the intrinsic RC $i$-band magnitude constructed from the clean RC sample. The yellow stars show the predicted model RC magnitudes corresponding to the same metallicities and ages that are used to predict the model RC colors shown in Figure 7. The red shaded and dashed lines present our default radial profile of the intrinsic RC magnitude to derive the distance between $2.7<\rho<8.5$ and outside this radial range, respectively.

the LMC disk. The effect of an unresolved temperature structure on the SFD98 map is especially severe in the central regions of the LMC disk where the LMC dust overwhelms the MW foreground. In the central regions, our reddening map shows an excellent morphological agreement with the highresolution Herschel dust emission images (Gordon et al. 2014).

The distribution of ratios between our reddening map and the SFD98 map can be described as a Gaussian profile with a peak around 1 when ignoring the central region (i.e., high-extinction regions), suggesting good quantitative agreement in the outer disk. This indicates that the majority of the reddening toward the non-central regions results from the MW foreground, which acts as a dust screen on stars behind it, and thus corroborates that the SFD98 map works well in the outer regions. If the LMC internal dust contributes to the majority of the reddening in the outer disk, one should expect discrepancy between the two maps. This is because the SFD98 map traces the total dust emission from a given region, whereas our map represents the median reddening values in each cell measured from individual RC stars that only sample dust in front of them. Thus, the SFD98 map would have shown systematically higher values than our map if internal LMC extinction dominated in the outer disk.

Figure 11 presents examples of CMDs dust-corrected by our map and the SFD98 map for low (Field 237), moderate (Field 49), and high (Field 41) extinction regions. In each case, we show an observed CMD, a CMD corrected using our RC-based reddening map, a CMD corrected using the emission-based SFD98 map, and distributions of $A_{i}$ values from our and SFD98 reddening maps. For the low-extinction case (top row), both our map and the SFD98 map return almost identical CMDs after correction, and their $A_{i}$ distributions are indeed very similar to each other, confirming that the SFD98 map can be used in low-extinction regions in the outer LMC disk. For the moderate extinction case, the CMD corrected by our map shows a tighter RGB sequence and a sharper SRC feature, while the CMD corrected by the SFD98 map shows the RC streak in the opposite direction and some artifacts in the MS because of overcorrection. The $A_{i}$ distribution from the SFD98 map clearly develops a long tail to larger values of $A_{i}$. As expected, the overcorrection problem with the SFD98 map becomes more severe in the high-extinction case, while our reddening map returns the CMD that seems reasonably well corrected overall.

As explained by SFD98 and discussed above, the SFD98 map fails in the optically bright LMC disk because the data on which the map was based could not resolve the temperature structure of the LMC disk. This failure explains the severe overcorrection of extinction in Fields 41 and 49 in Figure 11. 

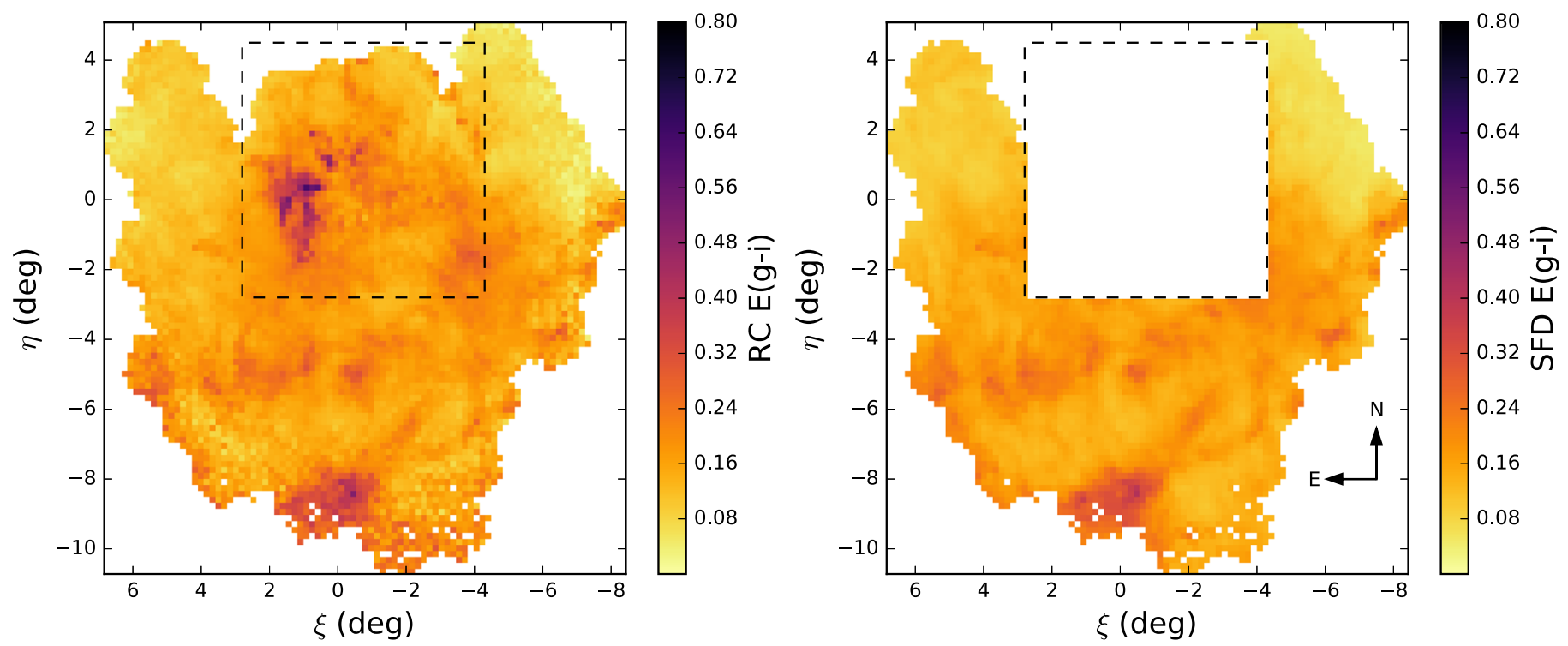

Figure 10. $E(g-i)$ reddening maps derived from our RC (left) and from the SFD98 $E(B-V)$ map (right). We use extinction coefficients for the $E(B-V)$ to $E(g-i)$ conversion that account for a systematic $\sim 14 \%$ overestimation in the SFD98 $E(B-V)$ map (see Schlafly \& Finkbeiner 2011; Abbott et al. 2018). A dashed box shows the inner region where the LMC emission dominates far-IR emission, and thus the SFD98 map cannot provide reliable measurements due to the lack of angular resolution to resolve complex temperature structures. Thus, we intentionally do not show their map inside the box. Outside the box, the two maps show an excellent agreement, both qualitatively as well as quantitatively, indicating that the majority of the extinction in the outer disk comes from the MW foreground cirrus. The electronic version of our reddening map is available as supplementary material to this paper. The data used to create this figure are available.

An additional issue is that SFD98 measured the total emission by dust, both foreground and internal to the LMC, along the line of sight, whereas our maps are sensitive to the total foreground extinction but only to the median of the LMC internal extinction. Thus, our map will either over- or undercorrect for the true extinction, depending on the relative geometry of individual stars and dust. This bias is likely to be most pronounced in the regions of high extinction found in the central LMC disk $\left(\rho<2^{\circ}\right)$. The extent of this bias will likely be no more than a few of tenths of magnitude as will be seen later in Figure 12. In regions of low to moderate internal LMC extinction (i.e., $\rho>2^{\circ}$ ), as shown in Figure 11, the bias should be negligible, as the Galactic foreground extinction makes up a larger fraction of the total extinction in these areas. Furthermore, the median relative distances between LMC subregions, corrected for dust using our map, would not significantly change in spite of the bias. Our use of the RC-derived extinction map is thus unlikely to affect the correct geometry of the galaxy midplane, and thus our results on the LMC's threedimensional structure should be robust against this extinction bias.

In Figure 12, we perform a cell-by-cell comparison of our median $A_{V}$ map and the $A_{V}$ map derived from the H I column density (Staveley-Smith et al. 2003) for a better quantitative verification of our reddening map in the inner star-forming disk where the internal LMC dust is dominant. The H I image used here was convolved to have a spatial resolution of $40^{\prime \prime}$ and removed a large-scale structured background emission by Gordon et al. (2014). We also construct our RC-based $A_{V}$ map at $40^{\prime \prime}$ resolution for a cell-by-cell comparison. For a given H I column density $\left(N_{\mathrm{HI}}\right)$, we obtain the optical extinction along a given sightline using the empirical relation obtained by correlating the reddening of stars with the $\mathrm{H}$ I column density in the LMC galaxy. A coefficient between the gas column density and extinction varies with the ISM properties (e.g., metallicity and ISM phase). For example, Fitzpatrick (1985) found the relation of $A_{V}=N_{\mathrm{H} \text { I }} /\left(7.74 \times 10^{21}\right)$ around the 30 Dor region. On the other hand, Gordon et al. (2003) measured the average LMC $A_{V}=N_{\mathrm{H} \mathrm{I}} /\left(3.25 \times 10^{21}\right)$, implying overall lower gas-todust ratios than the 30 Dor region.

In Figure 12, the underlying color scale shows the comparison RC-based $A_{V}$ values with $A_{V}$ values derived from the average LMC relation, while the red contour shows the comparison with $A_{V}$ values derived from the 30 Dor region relation. For both cases, our RC-based $A_{V}$ measurement correlates well with the H I -based $A_{V}$ measurement. This, combined with morphological agreement with the FIR dust emission images, suggests that our $\mathrm{RC}$ method works reasonably well in the inner LMC disk as well. Although these two specific coefficients do not result in one-toone correlation, it seems that the LMC might have a typical coefficient somewhere between these two coefficients, but determination of that coefficient or gas-to-dust ratio is beyond the scope of this paper. Besides the variation in gas-to-dust ratio across the LMC star-forming disk, another obvious thing that might contribute to the deviation from one-to-one correlation is the bias in our RC-based $A_{V}$ values especially at larger values of $A_{V}$ where the impact of difference between our median extinction and the total line-of-sight extinction toward individual stars is more significant. For the average LMC case, lower gas-to-dust ratios result in larger extinction values for a given $\mathrm{HI}$ column density than our RC-based median extinction values. On the other hand, for the 30 Dor case, our RC-based $A_{V}$ values are larger because larger gas-to-dust ratios lead to lower $A_{V}$ values for a given H I column density. These trends, shown in Figure 12, may in part be explained by the bias in our RC extinction map. The 1D histograms of $A_{V}$ values in Figure 12 show that most lines of sight have extinction less than $0.5 \mathrm{mag}$, indicating that the bias in our RC-based extinction map for the central starforming disk is likely no more than a few tenths of magnitude. 

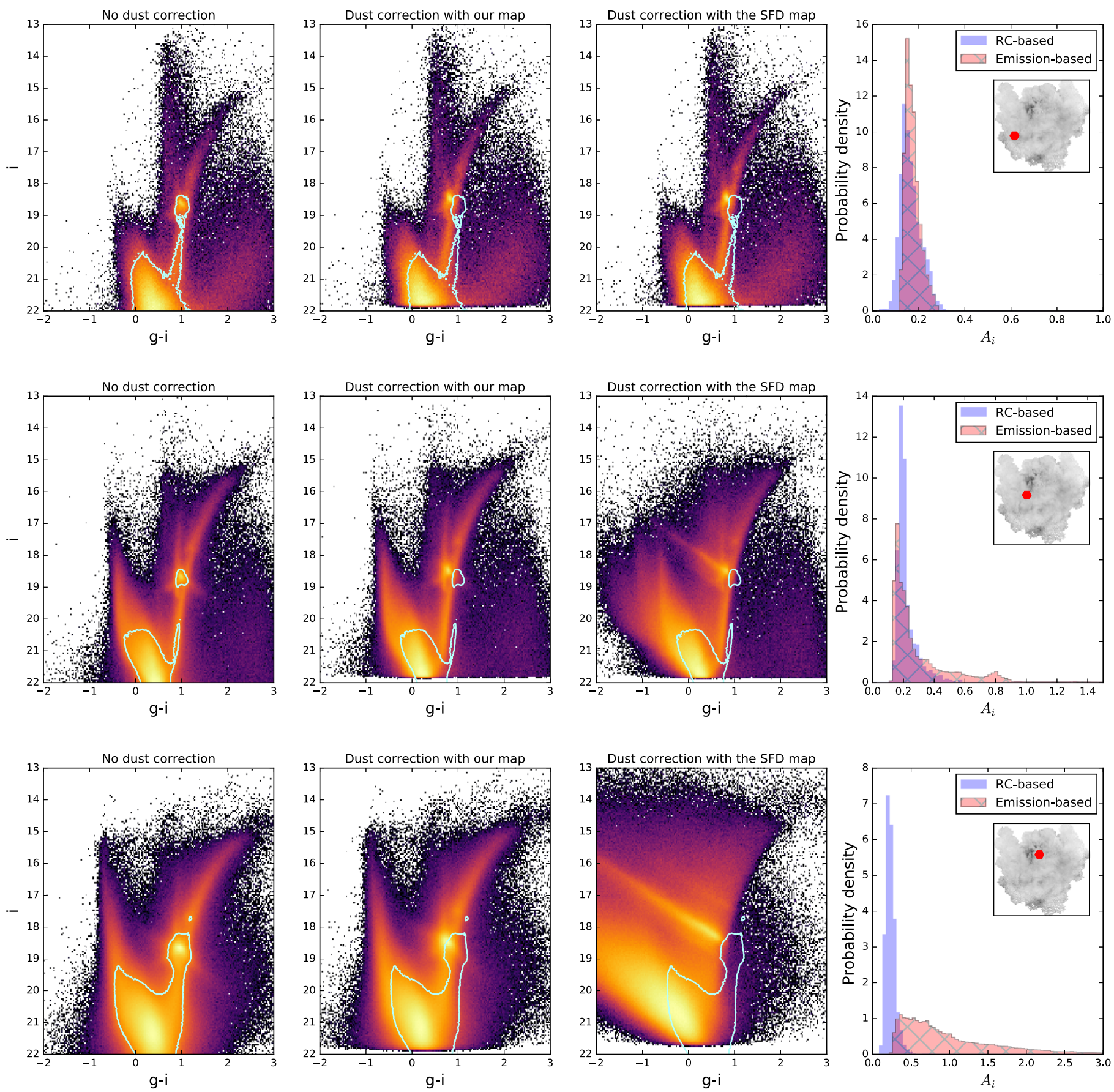

Figure 11. Examples for dust correction in low (Field 237), moderate (Field 49), and high (Field 41) extinction regions from top to bottom rows. In each row, we show an observed CMD (left), a CMD corrected using our RC-based reddening map (middle left), and a CMD corrected using the emission-based SFD98 map (middle right). To make comparison easy, we overplot the contours of an observed CMD in the corrected CMDs. In the low-extinction case, both our map and the SFD98 map work well and return almost identical CMDs after correction, confirming that the SFD98 map can be used in low-extinction regions in the outer LMC disk. In the moderate extinction case, the CMD corrected with our map shows a tighter RGB sequence and a sharper SRC feature while the CMD corrected with the SFD98 map starts suffering from overcorrection, resulting in the RC streak in the opposite direction and artifacts in the MS. As expected, the overcorrection problem gets worse in the high-extinction case, while our reddening map returns the CMD that seems reasonably well corrected. The last columns in each row show the $A_{i}$ distributions of each field from our and the SFD98 reddening maps. The inset panels show the locations of each field within the LMC (red hexagon). In the low-extinction region, two distributions are very similar to each other. However, in Fields 41 and 49, the distributions of $A_{i}$ from the SFD98 map feature long tails toward larger values of $A_{i}$. As discussed by SFD98 and in the text, the SFD98 maps do not trace extinction well in the inner LMC disk.

\section{A Three-dimensional Map of the LMC Disk}

\subsection{Inclination and Position Angle}

In this section, we constrain the LMC disk's $(i, \theta)$ using RC stars selected from the data covering a wide area of the unexplored southern part of the disk. First, we measure relative distances with respect to the LMC center using the extinctioncorrected relative $i$-band magnitudes for each subregion. With a distance to each subregion, the subregion positions are expressed in Cartesian coordinates $(X, Y, Z)$.

To characterize the properties of the LMC disk, we define a galaxy plane relative to the sky plane based on the 3D distribution 

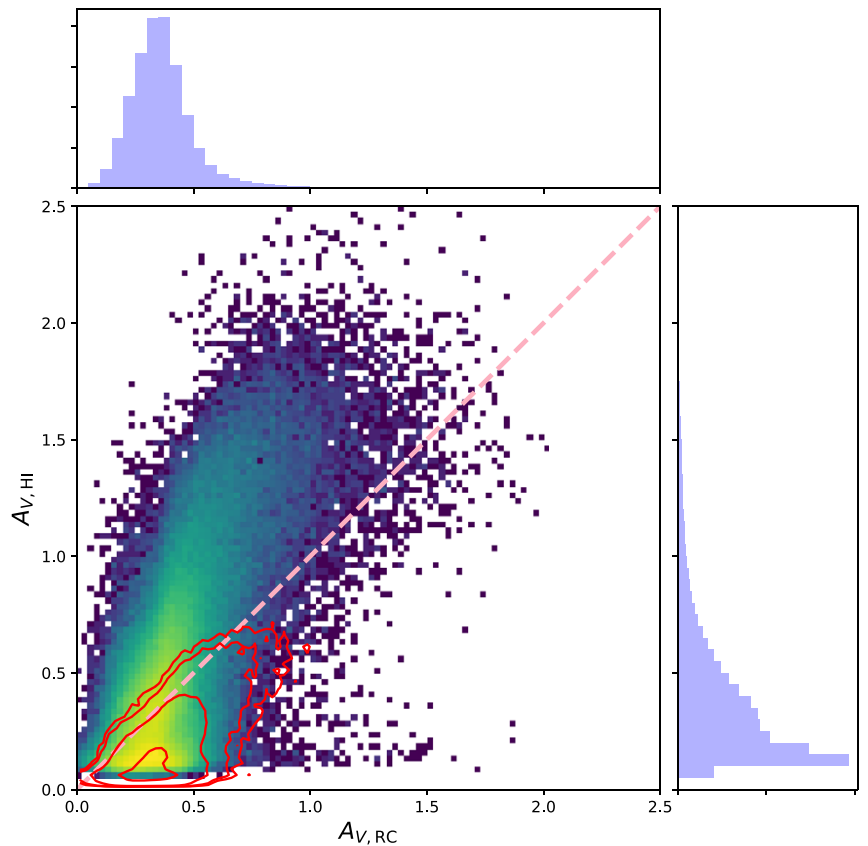

Figure 12. Cell-by-cell comparison between the mean $A_{V}$ map from our reddening map and the $A_{V}$ map derived from the $\mathrm{H}$ I column density $\left(N_{\mathrm{H}}\right)$ in the inner LMC disk. From $N_{\mathrm{H}}$, we determine $A_{V}$ using the average relation found in the LMC within $\sim 8 \%$ uncertainty (see Table 2 of Gordon et al. 2003). The red contour shows a cell-by-cell comparison with the $A_{V}$ values determined using the relation found around the 30 Dor region (Fitzpatrick 1985). For both cases, our $A_{V}$ values scale with the $A_{V}$ values derived from $N_{\mathrm{H} \text { I }}$, indicating our $\mathrm{RC}$-based reddening map traces dust in the inner LMC disk reasonably well. The pink dashed line corresponds to a one-to-one relationship. We also present the $1 \mathrm{D}$ histograms of the $A_{V}$ values to explicitly show that most of the sightlines have less than $0.5 \mathrm{mag}$ of extinction, indicating that the bias in our RC-based extinction map will likely be no more than a couple of tenths of magnitude.

of all cells by minimizing their distances to the plane using scipy.optimize.leastsq again. The unit normal vector $(\hat{n})$, defined in Cartesian coordinates, of the best-fit plane is $\left(n_{x}, n_{y}\right.$, $\left.n_{z}\right)=(0.375,-0.223,0.899)$. From this normal vector, we derive $i=\cos ^{-1}\left(n_{z}\right)$ and $\theta=\pi+\tan ^{-1}\left(n_{y} / n_{x}\right)$. The fitted plane has an inclination of $25^{\circ} .86 \pm 0.19$ and the position angle $(\theta)$ of $149^{\circ} .23 \pm 0.49$. The uncertainties reported here are random errors, associated with the plane fitting, calculated using the MCMC sampler emcee (Foreman-Mackey et al. 2013). We allow each of the 100 walkers to take 2000 steps and compute the $68 \%$ confidence interval as the $1 \sigma$ uncertainty of each fitting quantity after discarding the burn-in phase of the first 500 steps. These errors do not include any systematic uncertainties associated with stellar population effects. The systematic uncertainties in our $(i, \theta)$ measurements are provided in Section 7.

The LMC disk has been found to be twisted (varying line of nodes with galactic radius), warped (not a single plane), and flared (increasing scale height with galactic radius) likely due to tidal interactions with both the SMC and the MW (van der Marel \& Cioni 2001; Olsen \& Salyk 2002; Nikolaev et al. 2004; Subramanian \& Subramaniam 2009b; Balbinot et al. 2015). Therefore, the inclination $(i)$ and the position angle of the line of nodes $(\theta$; the intersection of the galaxy plane and the sky plane) depend on the spatial coverage of the survey data. Indeed, it has been shown that these two parameters vary with both angular distance from the galactic center and measurement techniques even for a given stellar tracer because of the complicated shape of the LMC disk (van der Marel \& Cioni 2001; Subramanian \& Subramaniam 2013;
Jacyszyn-Dobrzeniecka et al. 2017). Furthermore, it has been found that the determined LMC disk properties depend significantly on the choice of stellar populations to trace the disk structure (e.g., Balbinot et al. 2015).

In Figure 13, we explore the effect of twists and warps on $(i, \theta)$ measurement. We first define eight $1^{\circ}$ wide radial annuli between $2^{\circ}$ and $10^{\circ}$ on the tangent plane $(\xi, \eta)$, and then (1) measure $(i, \theta)$ from all $\mathrm{RC}$ cells in each annulus and (2) measure $(i, \theta)$ for all $\mathrm{RC}$ cells enclosed within a circular area that defines the outer edge of each annulus. Each annulus fitting reflects the local geometry better than the circular area fitting. Black dashed lines present how the $(i, \theta)$ measurements change for each annulus, while black solid lines show the dependence of $(i, \theta)$ on areal coverage (i.e., each circular area for a given angular distance from the LMC center). Like van der Marel \& Cioni (2001), we also find a strong radial dependence of $(i, \theta)$-in general, both $(i, \theta)$ rapidly (slowly) decrease with galactic radius in the inner (outer) disk. The inclination and position angle are large in the central region $\left(<4^{\circ}\right)$, where the bar is dominant. This indicates that the bar itself is likely tilted more than the rest of the disk (see Section 6.3). Inno et al. (2016) studied the 3D distribution of Cepheids and also found a strong radial dependence of $(i, \theta)$ in the inner LMC disk. The inclination angle from annulus fitting significantly increases in the very outer region $\left(>8^{\circ}\right)$, where the warp is dominant.

Both measuring methods (annulus versus circular area) produce similar radial trends, except for the outer region $\left(\rho>8^{\circ}\right)$. The inferred inclination angle dramatically decreases from $\sim 40^{\circ}$ to $\sim 25^{\circ}$ with angular distance until $\rho \simeq 7^{\circ}$ and then almost converges or slightly increases beyond that radius. On the other hand, the $\theta$ values are rather flat with a slight decrease after a sharp drop at $\rho<3.5-4.5$, ranging between $\sim 140^{\circ}$ and $\sim 160^{\circ}$. This trend is found in both measuring schemes, although the measured $\theta$ values from the annulus fitting are slightly smaller, and there is a drop to $\sim 120^{\circ}$ in the outer region.

We also investigate the radial dependence of $(i, \theta)$ values for the case in which a constant intrinsic RC color of 0.82 is used across the disk. The red lines in Figure 13 show $(i, \theta)$ results for the case of constant intrinsic RC color and circular area fitting. The inclination angles behave in an almost identical way to those from the radially varying intrinsic $\mathrm{RC}$ color case in all different areal coverages. There are only a few degree differences along the galactic radius. The position angles for the constant intrinsic RC color case also behave in a similar way to the radially varying intrinsic RC color case. For the $(i$, $\theta)$ measurements in each annulus, the constant color case follows almost the same pattern as the radially varying color case, and thus we do not overplot them for clarity.

For the LMC, its previously reported inclination angle ranges from $\sim 7^{\circ}$ to $\sim 40^{\circ}$, and its reported position angle also widely ranges from $\sim 100^{\circ}$ to $\sim 180^{\circ}$ (e.g., van der Marel \& Cioni 2001; Olsen \& Salyk 2002; Koerwer 2009; Subramanian \& Subramaniam 2010, 2013; Inno et al. 2016; JacyszynDobrzeniecka et al. 2017). Our measured $i$ and $\theta$ values, both from the best-fit plane and from the annulus/circular area fitting, fall well within these ranges. Specifically, the measured position angle is in good agreement with the reported values based on the RC (e.g., Olsen \& Salyk 2002). On the other hand, the measured $i$ is smaller than reported values based on the RC. This discrepancy from face-value comparison is likely due to our larger areal coverage of the southern disk than previous 

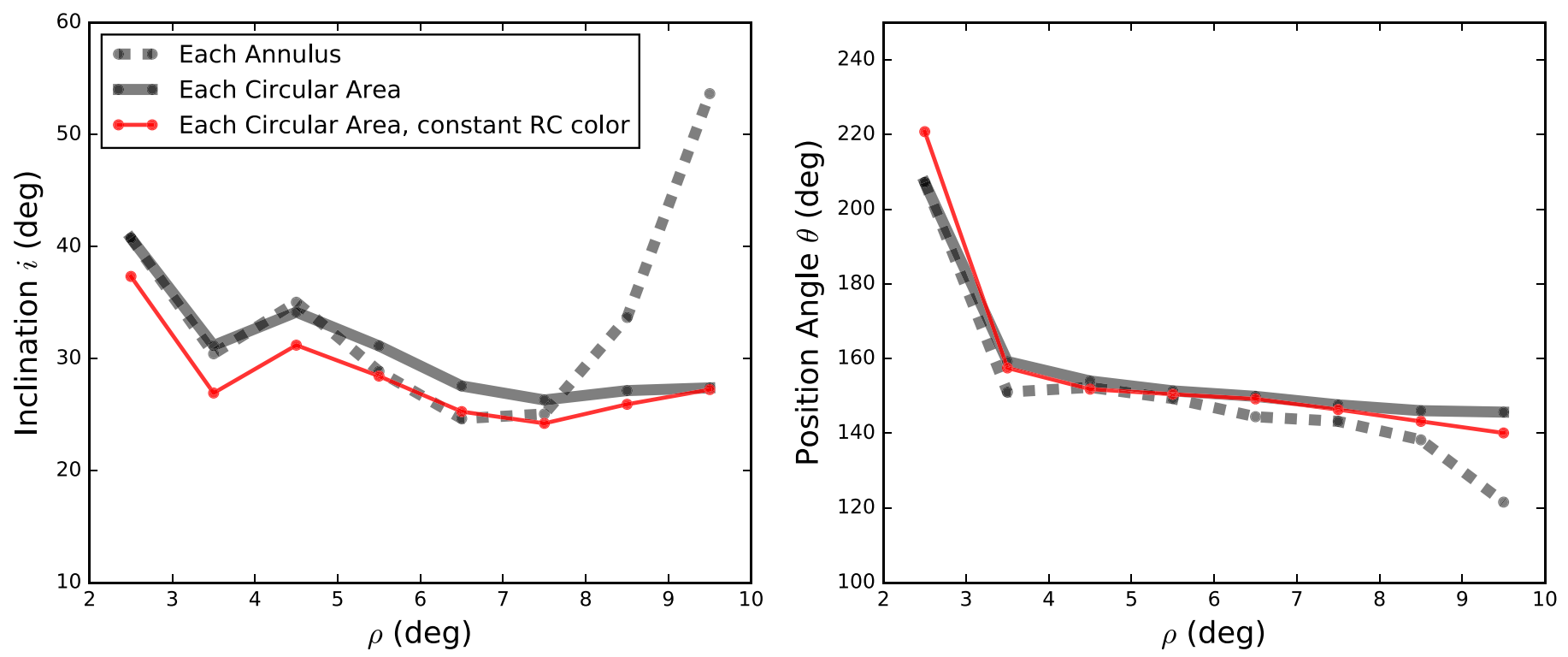

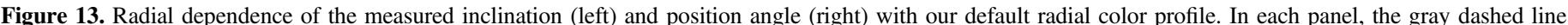

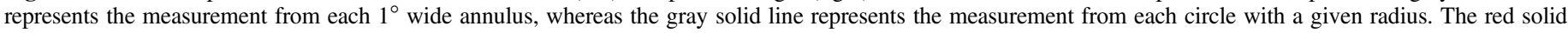

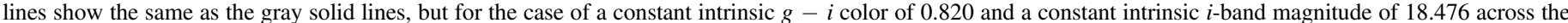

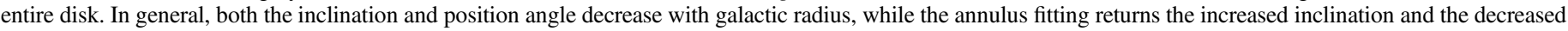
position angle in the two outer annuli where the outer warp appears.

studies. If we consider the smaller areal coverage of the different data sets based on Figure 13, the measured $i$ agrees well with the previous measurements. Our inclination is also similar to that measured in the northern outer disk by modeling the star number count map as an elliptical disk $\left(i=25^{\circ} .18 \pm 0.71\right.$; Mackey et al. 2016). Furthermore, our results are consistent with the measured $(i, \theta)$ based on combined information of line-of-sight velocities and proper motions (van der Marel \& Kallivayalil 2014). They measured $(i, \theta)=\left(30^{\circ} .3 \pm 5.9,153^{\circ} .7 \pm 5.4\right)$ for their young star sample (red super giant stars) and $\left(34^{\circ} .0 \pm 7.0,139^{\circ} .1 \pm 4.1\right)$ for their old star sample (a mix of carbon stars, AGB stars, and RGB stars).

\subsection{Disk Thickness}

We measure the thickness of the LMC's stellar disk from the distribution of distances of individual stars to the fitted plane. The distribution of distances to the fitted disk plane roughly follows a Gaussian distribution with a $\sigma$ of $\sim 1.0 \mathrm{kpc}$, leading to the FWHM thickness of $\sim 2.35 \mathrm{kpc}$. The LMC disk has been known to be thicker than the MW thick disk.

The line-of-sight depth of the LMC disk has been measured in the literature (e.g., Subramanian \& Subramaniam 2009b; Jacyszyn-Dobrzeniecka et al. 2017; Yanchulova Merica-Jones et al. 2017). We also measure a line-of-sight depth by looking at the distribution of individual stars along the $Z$-axis. We use stars only at $\rho>2^{\circ}$ to obtain a robust measurement by avoiding the artificial broadening along the line of sight due to the bias in our dust correction from using the median reddening values (see bottom panels in Figure 11). The measured line-ofsight depth is $\sim 7 \mathrm{kpc}$, which is larger than previous measurements by $1-2 \mathrm{kpc}$. This larger depth might be due to our larger areal coverage with the outer warp. As expected, including stars in the central region $\left(\rho<2^{\circ}\right)$ increases the lineof-sight depth by $\sim 0.5 \mathrm{kpc}$.

\subsection{Warp and Bar}

The left panel of Figure 14 shows the 3D structure of the LMC disk in projected Cartesian coordinates $(X, Y, Z)$ along the maximum distance gradient, which is perpendicular to the line of nodes. The grayscale reflects the stellar number counts along the viewing direction. The LMC disk is tilted against the sky plane, with the northeast (southwest) closer to (farther from) us. As shown, the LMC disk is clearly not a simple plane, which is consistent with previous studies-it has multiple ripples like the MW or other spiral galaxies (e.g., Gómez et al. 2013). Similar ripples in the inner region can be also found in the nearinfrared photometric data of the RC (Figure 9 in Subramanian \& Subramaniam 2013). A rippled disk is a common feature in a galaxy with close encounters with its satellite galaxies (Gómez et al. 2017).

In the LMC inner disk, Olsen \& Salyk (2002) detected a warp feature in the southwest (between $2^{\circ}$ and $4^{\circ}$ from the center along the maximum gradient). We reproduce their warp in our data as well by looking at cells corresponding to their observing fields (red circles Figure 14). These cells coincide with the highest stellar density. We mark their warp the "Olsen \& Salyk warp" in Figure 14, which turns out to be a portion of the rippled disk. Its amplitude $(\sim 2 \mathrm{kpc})$ and direction (toward us) are in excellent agreement with the findings in Olsen \& Salyk (2002). The Olsen \& Salyk warp corresponds to the feature seen between $-2.5 \mathrm{kpc}$ and $-4 \mathrm{kpc}$ along the maximum gradient. Its direction toward us makes the warp tilted opposite to the rest of the southwest disk. van der Marel \& Cioni (2001) suggested a warped disk based on decreasing inclination angle with angular radius between $2^{\circ} .7$ and $7^{\circ}$, indicating that the Olsen \& Salyk warp indeed affected their inclination and position angle measurements. We also detect such a decrease in inclination until $\sim 7^{\circ}$ from the center (see Figure 13). When measuring the disk inclination using the cells in the Olsen \& Salyk (2002) observing fields, but excluding the cells that form the Olsen \& Salyk warp, the fitting yields 

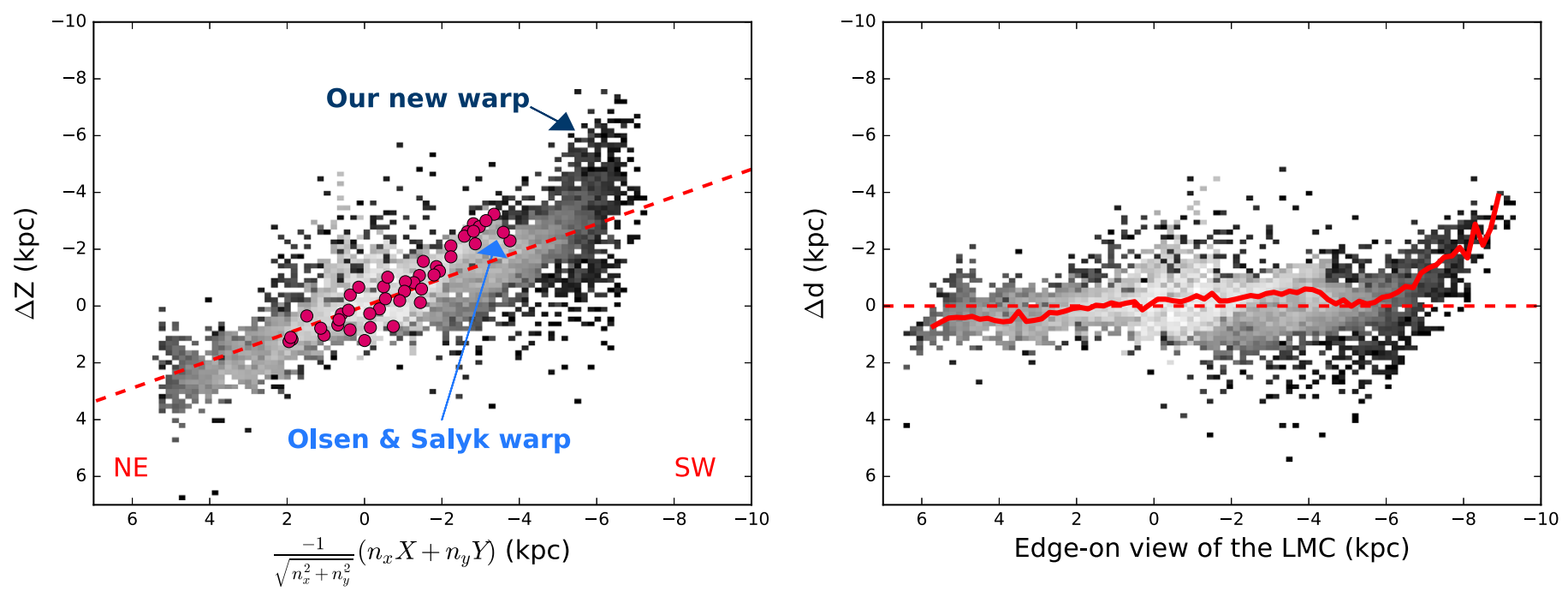

Figure 14. Left: the 3D RC distribution along the maximum line-of-sight depth gradient, which is perpendicular to the line of nodes. Positive (negative) $\Delta Z$ denotes closer (farther) to the observer than the galaxy center. The grayscale represents the star number counts- the brighter the pixel, the higher the stellar number density. The LMC disk is clearly tilted with respect to the sky plane $(Z=0)$ in a way that the NE is closer to us and the SW is farther away from us. We mark the warp found in the inner part of the disk by Olsen \& Salyk (2002) as well as the new warp in the outer disk toward the SMC. For clarity, we present the cells corresponding to the Olsen \& Salyk (2002) observing fields as red circles. Out of the 50 observing fields of Olsen \& Salyk (2002), 44 fields overlap with our data. Right: an edge-on view of the LMC disk. The $y$-axis is the distance from the fitted disk in this projection. The red solid line traces the median distance weighted by stellar number density.

$i=34^{\circ} .42$, which is consistent with $i=35^{\circ} .8 \pm 22^{\circ} .4$ measured by Olsen \& Salyk (2002).

In the right panel of Figure 14, we present the edge-on view of the disk. The red line shows the average distance to the fitted plane weighted by stellar number count in each $0.2 \mathrm{kpc}$ bin along the $x$-axis. With our large areal coverage for the southern disk, we find a new prominent warp in the southwest that starts at about $-5.5 \mathrm{kpc}$ from the center (or $\sim 7^{\circ}$ southwest from the center on the sky). The warp departs from the fitted disk plane up to $\sim 4 \mathrm{kpc}$ in a direction away from the Sun, which is in the opposite direction to the Olsen \& Salyk warp. This is the first detection of this outer warp, made possible by the large areal coverage of the SMASH data for the southern LMC disk. The warp feature is robust against the choice of the intrinsic RC color radial profile. Also, we do believe that the warp is not an artifact resulting from the edge of our observing area, which is imprinted as a sharp drop in stellar number density that is coincidentally parallel to the direction of the new warp. This is because the average distance from the galaxy plane starts to increase negatively (i.e., away from the observer) between -5 and $-6.5 \mathrm{kpc}$, where the effect of the observing edge does not kick in yet.

The bar stands out as a high density in the central region in Figure 4. The 3D spatial distribution of cells around the bar-it is non-trivial to identify only bar stars-shows a wide dispersion along the vertical direction (i.e., perpendicular to the plane). Thus, quantifying the bar structure as a plane is not reasonable. Instead, we can interpret the $\operatorname{larger}(i, \theta)$ of the inner disk $\left(\rho<4^{\circ}\right)$, which encompasses the bar, as evidence of a highly tilted bar. If the central bar significantly affects the determination of the geometry of the inner disk, the bar seems to be tilted relative to the fitted plane at least by $\sim 5^{\circ}-15^{\circ}$. Although the origin and the geometry of the off-centered bar in the LMC remains to be fully understood (e.g., Zhao \& Evans 2000; Zaritsky 2004; Bekki 2009; Subramaniam \& Subramanian 2009a), an off-centered and tilted bar naturally forms in Besla's Model 2 due to a recent direct collision with the SMC.

\section{Discussion}

\subsection{Implication for the LMC-SMC Interaction Histories}

The SMASH data enable us to constrain the 3D structure of the LMC disk and detect its interesting features. The LMC disk is found to be twisted and warped with ripples, and to have a tilted bar. These features were previously found in other studies, and are mainly associated with the inner LMC disk. With our SMASH data, which includes a large unexplored area of the southern disk, we also detect these features, confirming that the LMC is indeed significantly disturbed. Furthermore, we reveal a prominent warp in the outer southwest disk $\left(\rho>7^{\circ}\right)$ for the first time. This warp departs $\sim 4 \mathrm{kpc}$ from the LMC disk plane toward the SMC.

The current space velocities of the MCs favor their first infall into the MW halo, suggesting interactions with the SMC as the primary cause of the disturbed LMC disk rather than MW tides. Thus, morphological studies of the MCs are one of the keys to constraining their interaction history. Under the first infall scenario, Besla et al. (2012) investigated the role of tidal interactions between the MCs in their evolution using two different orbital histories between the MCs: (1) Model 1-the SMC completes two passages around the LMC without getting closer than $20 \mathrm{kpc}$, and (2) Model 2-the SMC completes three passages around the LMC including the recent ( $\sim 100-300 \mathrm{Myr}$ ago) direct collision. Each model successfully reproduces some properties of the Magellanic system, and while Model 1 better reproduces the large-scale gaseous structures, Model 2 performs significantly better at reproducing the detailed morphology and kinematics of the LMC disk.

The edge-on view in Figure 14 is comparable to the galaxy $y-z$ plane shown in the bottom panels of Figures 12 and 13 from Besla et al. (2012). For convenience, we show a modified version of an edge-on view stellar density map for Model 2 from Besla et al. (2012) in Figure 15. Their Model 2 clearly shows the outer warps in both the stellar and gaseous disks.

Although Besla et al. (2012) tried to connect their outer warp with the Olsen \& Salyk warp, which was the only prominent 


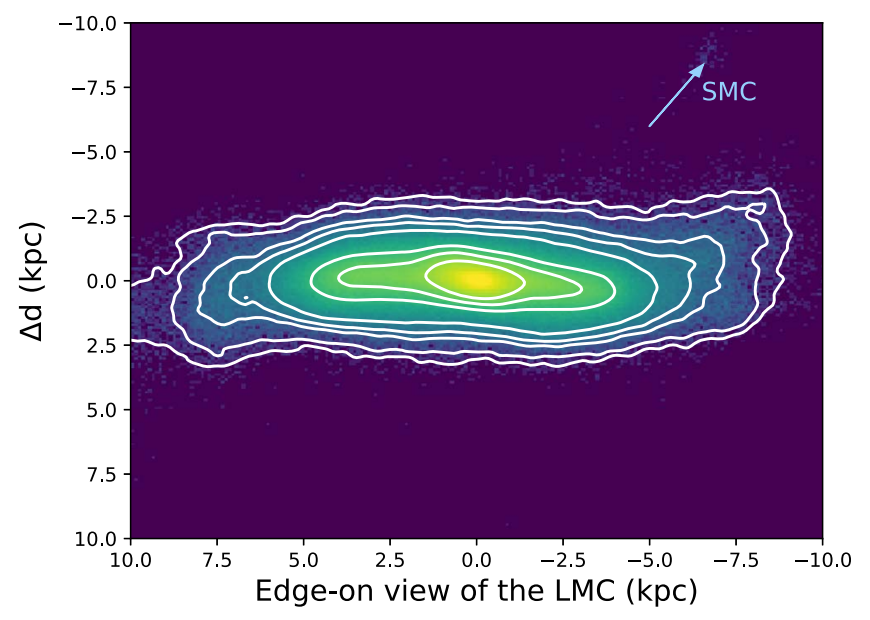

Figure 15. Modified version of the edge-on view of a stellar density map for Model 2 from Besla et al. (2012). This projection is comparable with our edgeon view shown in Figure 14. This model predicted the outer warp we detect for the first time in the outer disk toward the SMC due to the recent direct collision between the MCs a few hundred Myr ago. The direction toward the SMC is marked.

observed warp at that time, our study makes it clear now that the Olsen \& Salyk warp is one of the inner disk features. Thus, their predicted outer warp, starting at $\sim 5 \mathrm{kpc}$ from the disk center in their Model 2, actually has not had an observed counterpart until now. Based on the very similar position and size of the warp we find in the outer southwest disk, we argue that our new warp is the one corresponding to the outer warp presented in Model 2. Indeed, the position and amplitude of the warp is surprisingly similar to those predicted in their Model 2.

By contrast, the big warp in the outer disk does not appear in their Model 1, in which the SMC completed only two passages with large separations around the LMC. Because this outer warp is not created in Model 1, which has no direct collision between the MCs, our finding of the new warp (in addition to the tilted bar) supports a recent direct collision of the LMC with the SMC. The observed relative velocities of the SMC and LMC measured using new HST proper motions of the SMC (Zivick et al. 2018) further support this direct collision scenario.

More importantly, this new morphological feature might provide better constraints on model parameters for future theoretical modeling, not just for the MCs themselves but possibly also for other dwarf-dwarf pair systems (e.g., Stierwalt et al. 2015; Pearson et al. 2016). To provide a more complete picture of the outer warp, future studies should attempt to identify and quantify its counterpart in the northeast disk. If the warp originates owing to the tidal field of the MW, we expect an "integral" shape (e.g., Mackey et al. 2016; Gómez et al. 2017). If the structure originates from a collision with the SMC, the warp in the northeast is expected to be weaker than the outer warp we find (Besla et al. 2016).

\subsection{Effect of Variation in Stellar Populations}

Although the RC is a good probe of extinction and distance, it suffers from stellar population effects in its color and magnitude. In the previous section, we measured the radial profiles of the intrinsic color and magnitude using only stars from the clean $\mathrm{RC}$ regions across the LMC disk to take population variations into account as much as possible. This analysis uncovered a radial variation both in color and magnitude. However, the limited areal coverage $\left(2^{\circ} .7<\rho<\right.$ $8.5)$ of the clean RC regions prevents us from obtaining complete color and magnitude radial profiles that fully cover the innermost region $\left(\rho<2^{\circ} .7\right)$ to the outer region $\left(\rho>8^{\circ} .5\right)$.

The $(i, \theta)$ and amplitude of the warp might depend on our choice of the intrinsic RC color in the innermost and outermost radial bins. The amount of the extinction correction is determined by the choice of the intrinsic colors, and a larger extinction correction to the observed magnitude leads to a brighter intrinsic magnitude (i.e., closer to us), and vice versa. If we adopt a redder (bluer) intrinsic color for the outermost radial bin, then the distances to the regions associated with the warp feature would become larger (smaller), making the warp more (less) prominent. Furthermore, variations in the intrinsic magnitude in the innermost and outermost radial bins also affect the relative shape of the disk, probably leading to a change in the warp amplitude.

In this section, we attempt to adopt reasonable extremes of the assumed intrinsic color and magnitude for those regions based on both our own measurements in the rest of the radial range and the results of previous studies on the LMC's metallicity gradient and AMR. We discuss how our choice of the intrinsic color and magnitude in the central and outer regions can affect the $3 \mathrm{D}$ structural measurements. We also discuss the case for a single intrinsic color and magnitude across the disk to compare with the traditional RC method.

Case I: For the innermost radial bin $\left(\rho<2{ }^{\circ} 7\right)$, if we adopt $[\mathrm{Fe} / \mathrm{H}]=-0.4$ and $\mathrm{a} \sim 1 \mathrm{Gyr}^{\circ} \mathrm{old}^{24}$ population, the predicted mean $\mathrm{RC}$ color is $\sim 0.84$, which is redder than the rest of the disk, and the corresponding mean RC magnitude is $\sim 18.76 \mathrm{mag}$, which is $\sim 0.3 \mathrm{mag}$ fainter than the default magnitude in the innermost radial bin.

This fainter intrinsic magnitude in the innermost region induces a structure protruding from the disk by $4-5 \mathrm{kpc}$, making the outer warp slightly less prominent than the default case (by $\sim 10 \%$ ). For the main disk (i.e., excluding the protruding structure), we obtain the inclination of 26.56 and position angle of $147^{\circ} .02$ with respect to the results for our default case.

An elevated bar above the disk in the LMC has been suggested by some studies (e.g., Zhao \& Evans 2000; Nikolaev et al. 2004; Haschke et al. 2012). For example, Haschke et al. (2012) found evidence that the bar is $\sim 5 \mathrm{kpc}$ closer to us than the disk in their RR Lyrae sample, but no evidence was found in their Cepheid sample. On the other hand, JacyszynDobrzeniecka et al. (2017) interpreted the protruding structure seen in their OGLE-IV RR Lyrae stars as an artifact due to blending and crowding effects. In addition, Subramaniam \& Subramanian (2009a) analyzed the OGLE-III RC stars and concluded that the bar is located within the disk. We also do not take this protruding structure seriously because the inferred reddening from this color choice is lower than the previous measurements for the central region (e.g., Haschke et al. 2011; Tatton et al. 2013).

Although it might be reasonable to adopt this combination of age and metallicity for the innermost radial bin, the intrinsic RC color and magnitude vary significantly in the young age regime $(<1.5$ Gyr; see Figure 1 in Girardi \& Salaris 2001). This means that it is impossible to determine representative ages from our own clean RC sample without detailed SFH measurements in the central region. Stellar populations in the central region

\footnotetext{
${ }^{24}$ This is the young end for the mean RC age range (Girardi 2016).
} 
might be too complex to be described as one representative color and magnitude.

Case II: For the outermost radial bin $(\rho>8.5)$, if we extrapolate with the outer radial bin's slope of -0.033 dex $\operatorname{deg}^{-1}$, the expected color at $\rho=10^{\circ} .5$ is $\sim 0.7$. This color can be reasonably explained with a slightly lower metallicty $([\mathrm{Fe} / \mathrm{H}]=-0.9$ or -1.0$)$ and older age $(\sim 10 \mathrm{Gyr}$ or $\sim 8 \mathrm{Gyr})$ compared to the previous radial bin, which is also consistent with the LMC's smooth and shallow metallicity and age gradients. If we adopt the population of $[\mathrm{Fe} / \mathrm{H}]=-0.9$ and $10 \mathrm{Gyr}$, the corresponding model magnitude at $\rho=10^{\circ} .5$ is $\sim 18.6 \mathrm{mag}$, which requires a magnitude profile slope of $0.059 \mathrm{dex} \mathrm{deg}^{-1}$ in the outermost radial bin. With these modified color and magnitude radial profiles, we measure the inclination of $24^{\circ} .47$ and position angle of $155^{\circ} .66$. The outer warp becomes more prominent; its amplitude increases by $\sim 10 \%$ with respect to the results for our default case.

Case III: If we combine the changes in these two radial bins (i.e., Cases I and II), both the inclination and position angle are consistent with the results for our default case within uncertainties. This might be because the net effect from the bar region and the warp region compensate each other. The warp shows no noticeable change in its shape and amplitude.

Case IV: Adopting a constant intrinsic RC color and magnitude is the simplest way to derive line-of-sight extinctions and distances to RC stars. Previously, many studies measured the LMC structure in this way, and then discussed possible uncertainties due to population effects. If we assume no radial dependence of the intrinsic RC color and magnitude throughout our analysis, the inclination and position angle are $26.59\left(\sim 1^{\circ}\right.$ larger than the default case $)$ and $140^{\circ} .88\left(\sim 9^{\circ}\right.$ smaller than the default case). In this case, the Olsen \& Salyk warp becomes stronger, making the outer warp launch from below the fitted plane. This results in a decrease in an amplitude of the outer warp, which is measured from the fitted plane, by up to $\sim 25 \%$. If we make a fairer comparison with the other cases where the outer warp launches from the fitted plane by measuring its amplitude from where it actually starts, the amplitude is $\sim 10 \%$ smaller than the default case.

In conclusion, the systematic uncertainties due to population effects on the inclination are $-5.4 \% /+2.8 \%$ (corresponding to -1.39 and 0.73 ) and $-5.6 \% /+4.3 \%$ (corresponding to $-8^{\circ} .35$ and 6.43 ) on the position angle. The shape and the presence of the outer warp are robust against the choice of radial profiles of the intrinsic RC color and magnitude. The amplitude of the warp is marginally sensitive (up to $\sim 10 \%$ ) to the choice of the intrinsic color and magnitude in the innermost and outermost radial bins. Although there are small variations in the $(i, \theta)$ measurements with the population effects, the general trends of these parameters do not change: (1) the disk is tilted with respect to the sky plane (the northeast is closer to us, whereas the southwest is farther away from us), (2) the LMC has a welldeveloped warp toward the SMC in the southwestern region, and (3) the central bar is tilted relative to the rest of the disk. We also find that the disk thickness is robust against the variation in stellar populations (Cases I-IV) regardless of whether the central disk where our RC-derived median reddening map can cause the bias is included. The line-ofsight depth is also robust against the variation in stellar populations when excluding the protruding structure in the central region.

\section{Summary}

We use the high-quality SMASH data that map $\sim 480 \mathrm{deg}^{2}$ of the Magellanic System with high precision and accuracy both in photometry and astrometry. Out of 197 SMASH fields, we use 62 fields that cover the main body of the LMC $\left(\sim 5^{\circ}\right.$ to the north and $\sim 11^{\circ}$ to the south from the galactic center), and select 2.2 million RC stars to map the LMC's dust reddening and to understand its 3D structure. The SMASH data cover the southern periphery of the LMC disk, which has never been previously explored to this depth.

In the past, the RC has been extensively used to trace stellar structures because of its narrow color and magnitude ranges. However, its inherent dependence on stellar population (age and metallicity) has not been properly considered. For example, many studies used a constant color and magnitude assuming a single (or average) age and metallicity. In this study, we conduct a careful analysis to determine the intrinsic RC color and magnitude across the LMC disk.

To measure the intrinsic color and magnitude from our data, we first construct a clean RC sample by selecting stars in regions with negligible internal extinction (i.e., the presence of a clear separation between the main RC and the RGB). From this subsample, we measure the radial profile of the intrinsic color for the radial range $\left(2^{\circ} .7<\rho<8^{\circ} .5\right)$. The measured $\mathrm{RC}$ color radial profile shows a constant color over the middle part of the disk and bluer colors both for the inner and outer disks. Bluer color in the inner disk can be interpreted as the presence of metal-rich young populations, whereas bluer color in the outer disk can be interpreted as the presence of metal-poor old populations. As a default color profile, we adopt a constant color at $\rho=2.7$ and at $\rho=8.5$ for the innermost and the outermost radial bins, respectively.

We also investigate the radial dependence of the RC intrinsic magnitude using the clean sample after removing the inclination effect from the extinction-corrected observed magnitude. There is no significant radial change in the intrinsic magnitude between $4^{\circ}<\rho<8.5$ - only a slightly brighter magnitude in the inner region $\left(2.7<\rho<4^{\circ}\right)$ likely due to younger populations. As a default magnitude profile, we adopt a constant magnitude at $\rho=2.7$ and at $\rho=8.5$ for the innermost and the outermost radial bins, respectively.

After accounting for these population effects, we derive the reddening map that recovers the detailed features found from dust emission (SFD98) in the outer disk at an exquisite level and provides more reliable measurements in the inner disk. A cell-by-cell comparison shows that our reddening map scales with the inferred reddening map from the H I column density along the sightlines. The inner region where the SFD98 map fails shows high reddening values that likely result from LMC internal dust at a variety of temperatures, while the foreground MW dust seems to dominate most of the low-extinction regions outside this inner region.

We fit the LMC disk using the extinction-corrected $i$-band median magnitude map with $10^{\prime}$ by $10^{\prime}$ spatial resolution. The measured inclination and position angle with random uncertainties are $25^{\circ} .85 \pm 0.19$ and $149^{\circ} .23 \pm 0^{\circ} .49$, respectively. We also estimate the systematic uncertainties in our measurements due to additional population effects, which result from potential deviations from our default color and magnitude radial profile choice. Based on our choice of intrinsic colors for the innermost and outermost radial bins, the inclination varies from $24^{\circ} .47$ to $26^{\circ} .59$, and the position angle varies from $141^{\circ}$ 
to $153^{\circ}$. We also find a significant dependence of the inclination on the areal coverage of the data, which decreases with increasing areal coverage.

Finally, we detect a prominent warp in the southwestern disk that starts at $\rho \simeq 7^{\circ}$ with an amplitude of $\sim 4 \mathrm{kpc}$ toward the SMC direction. This warp is detected for the first time, and is different from the warp found in the inner disk by Olsen \& Salyk (2002), which we also detected in our data as a portion of the rippled disk. We also find that the cells around the bar show a broad vertical distribution. If we interpret the large inclination and position angles of the inner disk, which encompasses the bar, as a result of a tilted bar, the bar seems to deviate from the rest of the LMC disk by at least $\sim 5^{\circ}-15^{\circ}$. Both the warp and the tilted bar are consistent with those predicted in Model 2 from Besla et al. (2012). Thus, we suggest that the newly found warp and tilted bar are the product of a recent direct collision between the LMC and the SMC.

We are grateful to the referee for providing helpful comments to improve the paper. Y.C. and E.F.B. acknowledge support from NSF grant AST 1655677. M.-R.L.C. acknowledges support from the European Research Council (ERC) under the European Union's Horizon 2020 research and innovation program (grant agreement No. 682115). A.M. acknowledges partial support from CONICYT FONDECYT regular 1181797. B.C.C. acknowledges the support of the Australian Research Council through Discovery project DP150100862. T.D.B. acknowledges support from the European Research Council (ERC StG-335936). D.M.D. acknowledges support by Sonderforschungsbereich (SFB) 881 "The Milky Way System" of the German Research Foundation (DFG), particularly through subprojects A2. Based on observations at Cerro Tololo Inter-American Observatory, National Optical Astronomy Observatory (NOAO Prop. IDs: 2013A-0411 and 2013B-0440; PI: Nidever), which is operated by the Association of Universities for Research in Astronomy (AURA) under a cooperative agreement with the National Science Foundation. This project used data obtained with the Dark Energy Camera (DECam), which was constructed by the Dark Energy Survey (DES) collaboration. Funding for the DES Projects has been provided by the U.S. Department of Energy, the U.S. National Science Foundation, the Ministry of Science and Education of Spain, the Science and Technology Facilities Council of the United Kingdom, the Higher Education Funding Council for England, the National Center for Supercomputing Applications at the University of Illinois at Urbana-Champaign, the Kavli Institute of Cosmological Physics at the University of Chicago, Center for Cosmology and Astro-Particle Physics at the Ohio State University, the Mitchell Institute for Fundamental Physics and Astronomy at Texas A\&M University, Financiadora de Estudos e Projetos, Fundação Carlos Chagas Filho de Amparo, Financiadora de Estudos e Projetos, Fundação Carlos Chagas Filho de Amparo à Pesquisa do Estado do Rio de Janeiro, Conselho Nacional de Desenvolvimento Cientifico e Tecnológico and the Ministério da Ciência, Tecnologia e Inovação, the Deutsche Forschungsgemeinschaft and the Collaborating Institutions in the Dark Energy Survey. The Collaborating Institutions are Argonne National Laboratory, the University of California at Santa Cruz, the University of Cambridge, Centro de Investigaciones Enérgeticas, Medioambientales y Tecnológicas-Madrid, the University of Chicago, University College London, the DESBrazil Consortium, the University of Edinburgh, the Eidgenössische Technische Hochschule (ETH) Zürich, Fermi National
Accelerator Laboratory, the University of Illinois at UrbanaChampaign, the Institut de Ciències de l'Espai (IEEC/CSIC), the Institut de Física d'Altes Energies, Lawrence Berkeley National Laboratory, the Ludwig-Maximilians Universität München and the associated Excellence Cluster Universe, the University of Michigan, the National Optical Astronomy Observatory, the University of Nottingham, the Ohio State University, the University of Pennsylvania, the University of Portsmouth, SLAC National Accelerator Laboratory, Stanford University, the University of Sussex, and Texas A\&M University.

Facility: Blanco (DECam).

Software: scipy (Jones et al. 2001), numpy (van der Walt et al. 2011), matplotlib (Hunter 2007), ipython (Pérez \& Granger 2007), astropy (The Astropy Collaboration et al. 2018), emcee (Foreman-Mackey et al. 2013).

\section{ORCID iDs}

Yumi Choi (1D) https://orcid.org/0000-0003-1680-1884 David L. Nidever (1) https://orcid.org/0000-0002-1793-3689

Knut Olsen (iD https://orcid.org/0000-0002-7134-8296 Robert D. Blum (1) https://orcid.org/0000-0002-8622-4237 Dennis Zaritsky (1) https://orcid.org/0000-0002-5177-727X Roeland P. van der Marel (ib https://orcid.org/0000-00017827-7825

Eric F. Bell (ib https://orcid.org/0000-0002-5564-9873 Carme Gallart (i) https://orcid.org/0000-0001-6728-806X Maria-Rosa L. Cioni (i) https://orcid.org/0000-0002-6797-696X L. Clifton Johnson (1D https://orcid.org/0000-0001-6421-0953 A. Katherina Vivas (iD https://orcid.org/0000-0003-4341-6172 Abhijit Saha (10 https://orcid.org/0000-0002-6839-4881 Antonela Monachesi (i) https://orcid.org/0000-0003-2325-9616

Blair C. Conn (1D https://orcid.org/0000-0001-6959-4546 David Martinez-Delgado (iD https://orcid.org/0000-00033835-2231

Guy S. Stringfellow (iD https://orcid.org/0000-0003-1479-3059

\section{References}

Abbott, T. M. C., Abdalla, F. B., Annis, J., et al. 2018, MNRAS, 480, 3879 Balbinot, E., Santiago, B. X., Girardi, L., et al. 2015, MNRAS, 449, 1129 Bekki, K. 2009, MNRAS, 393, L60

Besla, G., Kallivayalil, N., Hernquist, L., et al. 2007, ApJ, 668, 949 Besla, G., Kallivayalil, N., Hernquist, L., et al. 2012, MNRAS, 421, 2109 Besla, G., Martínez-Delgado, D., van der Marel, R. P., et al. 2016, ApJ, 825, 20 Bovy, J., Nidever, D. L., Rix, H.-W., et al. 2014, ApJ, 790, 127 Bressan, A., Marigo, P., Girardi, L., et al. 2012, MNRAS, 427, 127 Caldwell, J. A. R., \& Coulson, I. M. 1986, MNRAS, 218, 223 Carrera, R., Conn, B. C., Noël, N. E. D., Read, J. I., \& López Sánchez, Á. R. 2017, MNRAS, 471, 4571

Carrera, R., Gallart, C., Aparicio, A., \& Hardy, E. 2011, AJ, 142, 61 Carrera, R., Gallart, C., Hardy, E., Aparicio, A., \& Zinn, R. 2008, AJ, 135, 836 Castellani, V., Degl'Innocenti, S., Girardi, L., et al. 2000, A\&A, 354, 150 Chambers, K. C., Magnier, E. A., Metcalfe, N., et al. 2016, arXiv:1612.05560 Choi, Y., Nidever, D. L., Olsen, K., et al. 2018, arXiv:1805.00481 Choudhury, S., Subramaniam, A., \& Cole, A. A. 2016, MNRAS, 455, 1855 Connors, T. W., Kawata, D., \& Gibson, B. K. 2006, MNRAS, 371, 108 Connors, T. W., Kawata, D., Maddison, S. T., \& Gibson, B. K. 2004, PASA, 21,222

Dalcanton, J. J., Fouesneau, M., Hogg, D. W., et al. 2015, ApJ, 814, 3 de Grijs, R., Wicker, J. E., \& Bono, G. 2014, AJ, 147, 122

de Vaucouleurs, G., \& Freeman, K. C. 1972, VA, 14, 163

Epchtein, N., de Batz, B., Capoani, L., et al. 1997, Msngr, 87, 27

Feast, M. W., Abedigamba, O. P., \& Whitelock, P. A. 2010, MNRAS, 408, L76

Fitzpatrick, E. L. 1985, ApJ, 299, 219

Fitzpatrick, M. J., Graham, M. J., Mighell, K. J., et al. 2016, Proc. SPIE, 9913, 99130L

Flaugher, B., Diehl, H. T., Honscheid, K., et al. 2015, AJ, 150, 150 
Foreman-Mackey, D., Hogg, D. W., Lang, D., \& Goodman, J. 2013, PASP, 125,306

Gaia Collaboration, Brown, A. G. A., Vallenari, A., et al. 2016, A\&A, 595, A2

Gallart, C., Stetson, P. B., Meschin, I. P., Pont, F., \& Hardy, E. 2008, ApJL, 682, L89

Gardiner, L. T., \& Noguchi, M. 1996, MNRAS, 278, 191

Girardi, L. 2016, ARA\&A, 54, 95

Girardi, L., \& Salaris, M. 2001, MNRAS, 323, 109

Gómez, F. A., Minchev, I., O’Shea, B. W., et al. 2013, MNRAS, 429, 159

Gómez, F. A., White, S. D. M., Grand, R. J. J., et al. 2017, MNRAS, 465, 3446

Gordon, K. D., Clayton, G. C., Misselt, K. A., Landolt, A. U., \& Wolff, M. J. 2003, ApJ, 594, 279

Gordon, K. D., Roman-Duval, J., Bot, C., et al. 2014, ApJ, 797, 85

Harris, J., \& Zaritsky, D. 2009, AJ, 138, 1243

Haschke, R., Grebel, E. K., \& Duffau, S. 2011, AJ, 141, 158

Haschke, R., Grebel, E. K., \& Duffau, S. 2012, AJ, 144, 106

Hunter, J. D. 2007, CSE, 9, 90

Inno, L., Bono, G., Matsunaga, N., et al. 2016, ApJ, 832, 176

Jacyszyn-Dobrzeniecka, A. M., Skowron, D. M., Mróz, P., et al. 2017, AcA, 67,1

Jones, E., Oliphant, T., Peterson, P., et al. 2001, SciPy: Open Source Scientific Tools for Python, http://www.scipy.org/

Kallivayalil, N., van der Marel, R. P., \& Alcock, C. 2006a, ApJ, 652, 1213

Kallivayalil, N., van der Marel, R. P., Alcock, C., et al. 2006b, ApJ, 638, 772

Kallivayalil, N., van der Marel, R. P., Besla, G., Anderson, J., \& Alcock, C. 2013, ApJ, 764, 161

Kim, S., Staveley-Smith, L., Dopita, M. A., et al. 1998, ApJ, 503, 674

Koerwer, J. F. 2009, AJ, 138, 1

Mackey, A. D., Koposov, S. E., Erkal, D., et al. 2016, MNRAS, 459, 239

Majewski, S. R., Zasowski, G., \& Nidever, D. L. 2011, ApJ, 739, 25

Marigo, P., Girardi, L., Bressan, A., et al. 2017, ApJ, 835, 77

McClure-Griffiths, N. M., Pisano, D. J., Calabretta, M. R., et al. 2009, ApJS, 181,398

Meschin, I., Gallart, C., Aparicio, A., et al. 2014, MNRAS, 438, 1067

Monteagudo, L., Gallart, C., Monelli, M., Bernard, E. J., \& Stetson, P. B. 2018, MNRAS, 473, L16

Nidever, D. L., Bovy, J., Bird, J. C., et al. 2014, ApJ, 796, 38

Nidever, D. L., Olsen, K., Walker, A. R., et al. 2017, AJ, 154, 199

Nikolaev, S., Drake, A. J., Keller, S. C., et al. 2004, ApJ, 601, 260

Noël, N. E. D., Conn, B. C., Carrera, R., et al. 2013, ApJ, 768, 109

Olsen, K. A. G., \& Salyk, C. 2002, AJ, 124, 2045
Olsen, K. A. G., Zaritsky, D., Blum, R. D., Boyer, M. L., \& Gordon, K. D. 2011, ApJ, 737, 29

Pearson, S., Besla, G., Putman, M. E., et al. 2016, MNRAS, 459, 1827

Pérez, F., \& Granger, B. E. 2007, CSE, 9, 21

Piatek, S., Pryor, C., \& Olszewski, E. W. 2008, AJ, 135, 1024

Piatti, A. E., \& Geisler, D. 2013, AJ, 145, 17

Pieres, A., Santiago, B., Balbinot, E., et al. 2016, MNRAS, 461, 519

Saha, A., Olszewski, E. W., Brondel, B., et al. 2010, AJ, 140, 1719

Schlafly, E. F., \& Finkbeiner, D. P. 2011, ApJ, 737, 103

Schlegel, D. J., Finkbeiner, D. P., \& Davis, M. 1998, ApJ, 500, 525

Skrutskie, M. F., Cutri, R. M., Stiening, R., et al. 2006, AJ, 131, 1163

Stanek, K. Z., \& Garnavich, P. M. 1998, ApJL, 503, L131

Staveley-Smith, L., Kim, S., Calabretta, M. R., Haynes, R. F., \& Kesteven, M. J. 2003, MNRAS, 339, 87

Stetson, P. B. 1987, PASP, 99, 191

Stierwalt, S., Besla, G., Patton, D., et al. 2015, ApJ, 805, 2

Subramaniam, A., \& Subramanian, S. 2009a, ApJL, 703, L37

Subramanian, S., \& Subramaniam, A. 2009b, A\&A, 496, 399

Subramanian, S., \& Subramaniam, A. 2010, A\&A, 520, A24

Subramanian, S., \& Subramaniam, A. 2013, A\&A, 552, A144

Tatton, B. L., van Loon, J. T., Cioni, M.-R., et al. 2013, A\&A, 554, A33

The Astropy Collaboration, Price-Whelan, A. M., Sipőcz, B. M., et al. 2018, AJ, 156, 123

Valdes, F., Gruendl, R. \& DES Project 2014, in ASP Conf. Ser. 485, Astronomical Data Analysis Software and Systems XXIII, ed. N. Manset \& P. Forshay (San Francisco, CA: ASP), 379

van der Marel, R. P. 2001, AJ, 122, 1827

van der Marel, R. P., Alves, D. R., Hardy, E., \& Suntzeff, N. B. 2002, AJ, 124, 2639

van der Marel, R. P., \& Cioni, M.-R. L. 2001, AJ, 122, 1807

van der Marel, R. P., \& Kallivayalil, N. 2014, ApJ, 781, 121

van der Walt, S., Colbert, S. C., \& Varoquaux, G. 2011, CSE, 13, 22

Weisz, D. R., Dolphin, A. E., Skillman, E. D., et al. 2013, MNRAS, 431, 364

Yanchulova Merica-Jones, P., Sandstrom, K. M., Johnson, L. C., et al. 2017, ApJ, 847, 102

York, D. G., Adelman, J., Anderson, J. E., Jr., et al. 2000, AJ, 120, 1579

Yoshizawa, A. M., \& Noguchi, M. 2003, MNRAS, 339, 1135

Zaritsky, D. 2004, ApJL, 614, L37

Zaritsky, D., Harris, J., Thompson, I. B., \& Grebel, E. K. 2004, AJ, 128, 1606

Zhao, H., \& Evans, N. W. 2000, ApJL, 545, L35

Zivick, P., Kallivayalil, N., van der Marel, R. P., et al. 2018, ApJ, 864, 55 